\title{
HOMOGENIZATION OF MANY-BODY STRUCTURES SUBJECT TO LARGE DEFORMATIONS
}

\author{
Philipp Emanuel Stelzig ${ }^{1,2}$
}

\begin{abstract}
We give a first contribution to the homogenization of many-body structures that are exposed to large deformations and obey the noninterpenetration constraint. The many-body structures considered here resemble cord-belts like they are used to reinforce pneumatic tires. We establish and analyze an idealized model for such many-body structures in which the subbodies are assumed to be hyperelastic with a polyconvex energy density and shall exhibit an initial brittle bond with their neighbors. Noninterpenetration of matter is taken into account by the Ciarlet-Nečas condition and we demand deformations to preserve the local orientation. By studying $\Gamma$-convergence of the corresponding total energies as the subbodies become smaller and smaller, we find that the homogenization limits allow for deformations of class special functions of bounded variation while the aforementioned kinematic constraints are conserved. Depending on the many-body structures' geometries, the homogenization limits feature new mechanical effects ranging from anisotropy to additional kinematic constraints. Furthermore, we introduce the concept of predeformations in order to provide approximations for special functions of bounded variation while preserving the natural kinematic constraints of geometrically nonlinear solid mechanics.
\end{abstract}

Mathematics Subject Classification. 74Q05, 74B20, 74R10, 74M15, 74G65, 35B27, 49J45.

Received February 1, 2010. Revised July 20, 2010.

Published online December 23, 2010.

\section{INTRODUCTION}

In this article we rigorously derive effective mathematical models that describe the mechanics of certain many-body structures being exposed to large deformations. In our use of language, many-body structures are composed of a very large number of identical elastic (sub-)bodies which may come into mechanical contact, but may not interpenetrate each other. In the sequel we will abbreviate the term "many-body structure" by MBS. Indeed, MBSs in our sense are omnipresent in daily life and engineering applications alike. Examples include fibre-composed objects like textiles, ropes or cables, materials featuring reinforcing inclusions like fibrecomposites or reinforced concrete, but also brick walls. All larger organic objects are formed by a vast number of more or less identical cells, thus are MBSs in our sense, too.

\footnotetext{
Keywords and phrases. Homogenization, large deformations, contact mechanics, noninterpenetration, many-body structure, cord-belt, polyconvexity, brittle fracture, $\Gamma$-convergence.

${ }^{1}$ Lehrstuhl für numerische Mathematik und Steuerungstheorie, Zentrum Mathematik, Technische Universität München, Boltzmannstrasse 3, 85748 Garching bei München, Germany. stelzig@ma.tum.de

2 Dipartimento di Matematica, Università degli Studi di Trento, via Sommarive 14, 38050 Povo (TN), Italy.
} 

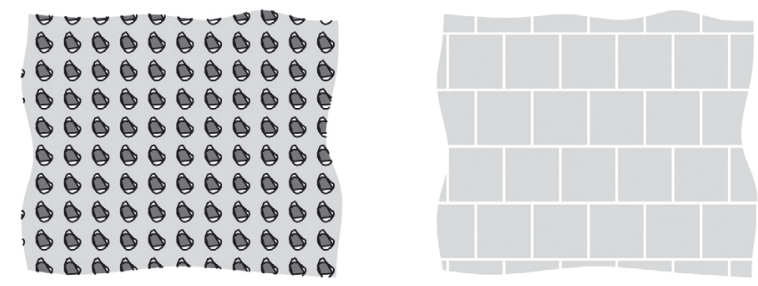

Figure 1. Matrix-inclusion type (left) and free MBSs (right).

In applications, MBSs are mostly regarded as one single object. This is due to the fact that their relevant length scale in applications is far larger than the characteristic size of the MBSs' subbodies. A such-like simplification is also desirable from the viewpoint of numerics: MBSs can consist of thousands of subbodies which may come into mechanical contact. Here we advice the reader that the mechanical nature of MBSs derives exclusively from contact mechanisms, the most important being noninterpenetration of matter. Hence, it is crucial to include a noninterpenetration-condition in any reasonable model of MBSs. Yet, contact problems are very delicate to deal with in a numerical treatment. Therefore, one is naturally interested in models for MBSs that do not pay attention to every possible contact problem on the scale of the subbodies.

A method to reduce a MBS to one single object on the relevant length scale (the "macroscale") is to average out in whatever sense the scale of the subbodies (the "microscale"). Eliminating the small scale of a MBS also avoids treating all the possible contact problems between the subbodies involved. The mathematically natural way to do so appears to be homogenization: one starts with a mathematical model for a MBS that accounts for all subbodies and noninterpenetration and then studies the asymptotics of the model as the characteristic size of the subbodies vanishes, i.e. as the MBS composes of more and more bodies.

Before giving an outline of our work and the results obtained we include a short review of the various different modelings and homogenization results for MBSs.

\section{A short literature review}

Real-life MBSs can be roughly classified into two categories (see Fig. 1). The first category contains what we call the matrix-inclusion type MBS. Therein, a connected matrix material separates the subbodies of the MBS from each other and assigns them the role of inclusions. This is the case for reinforced materials like e.g. fibre-reinforced composites. Whereas the second category contains all those MBSs in which there is no matrix material separating the subbodies. Instead, the subbodies are in contact with each other and can move freely as long as they do not interpenetrate. This is why we refer to the second type as free MBSs. Examples for free MBSs include the mentioned textiles, ropes, brick walls and laminated MBSs. We advice the reader that laminated MBSs, i.e. MBSs in which the subbodies exhibit an initial breakable bond with their neighbors, can undergo exactly the same deformations like their non-laminated counterparts.

A major difference between the two types of MBSs is that the matrix-inclusion type behaves often far more regular than free MBSs. Both types allow for discontinuities in the deformations on the scale of the subbodies across contacting boundaries. In the case of free MBSs such microscopic discontinuities can obviously join and lead to discontinuities on the MBSs' macroscale. In matrix-inclusion type MBSs on the other hand, microscopic discontinuities cannot extend into the matrix material. Provided the volume fraction of the matrix material is sufficiently large (say constant), the microscopic discontinuities in matrix-inclusion type MBSs cannot join, thus there are no macroscopic cracks. This regularizing effect has been observed in a number of works dealing with the homogenization of periodic matrix-inclusion type MBSs in a geometrically and constitutively linear setting, cf. $[16,19]$. However, if the volume fraction of the matrix material is too small, then microscopic discontinuities may still lead to macroscopic cracks. In this case we refer the reader to the recent results [3,12] and also to $[21,22]$. The homogenization of free MBSs has gained very little attention in the mathematical literature so far, the only reference known to us being [5]. Therein, the authors homogenize a periodic, geometrically and 

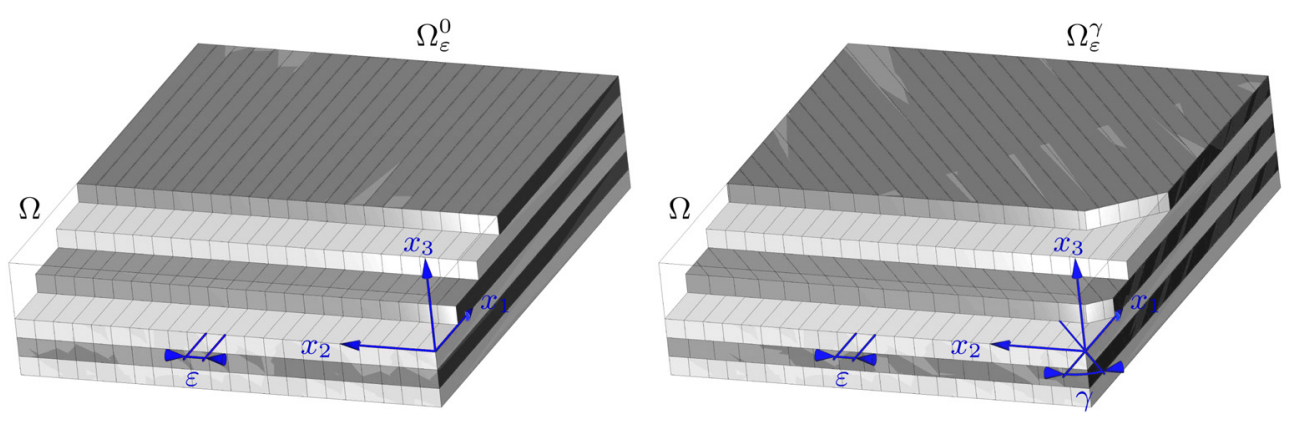

Figure 2. Cord-belt like MBSs with zero (left) and nonzero cord-angle $\gamma$ (right).

constitutively linear MBS in which the subbodies are not glued together and leave no empty space between each other (e.g. a brick wall without mortar between the bricks). As expected, they find the homogenization limit to allow for highly irregular deformations of class BD, the functions of bounded deformation.

In the regime of large deformations there are no homogenization results for MBSs available yet, neither for matrix-inclusion type MBSs nor for free MBSs. One of the reasons that renders the mathematics of MBSs far more delicate in the geometrically nonlinear regime is the noninterpenetration constraint. For small deformations one expects the subbodies of a MBS to be displaced only marginally, the same goes for their potential contact boundaries. Consequently, noninterpenetration of matter in the small deformation regime is accounted for by a local condition imposed on potential contact boundaries, the so-called Signorini boundary condition [17,23]. For large deformations instead, one has to impose a nonlocal, global condition of noninterpenetration of matter, i.e. injectivity of the deformations. Goal of the present work is to provide a first homogenization result for free MBSs in the geometrically and constitutively nonlinear regime.

\section{This work's matter}

Starting point for our studies was an application in tire reinforcement technology (see [24] for motivation). Modern pneumatic tires derive their outer shape and mechanical stability mostly from reinforcements embedded into the rubber matrix. An introduction to the various structural elements and different designs of pneumatic tires can e.g. be found in [25] or in product releases of the tire manufacturers. The most vital reinforcements found in pneumatic tires like the carcass plies or the belt are layered reinforcements made from cords. Cords are thin rope-like objects made from a small number of usually steel- or nylon-wires. In these reinforcements - which we call cord-belts in the sequel - a single cord-layer consists of parallel straight cords lying right next to each other, while the whole structure is composed of several cord-layers piled up. In applications one encounters two different designs of cord-belts. In the first design the orientations of the cords are equal in all layers (featured in the carcass plies of radial-ply tires). Whereas in the second design the orientations of the cords alternate in adjacent layers by the cord-angle (featured in the carcass plies of bias-ply tires and often also in the belt). Like the cord-angle, the number of layers in a cord-belt strongly depends on the scope of use: carcass plies of heavy load tires or aircraft tires may consist of as many as 20 layers.

We aim to analyze how the fact of being composed of a large number of slender elastic bodies enters the mechanical response of MBSs with cord-belt like geometry to large deformations.

We consider MBSs $\Omega_{\varepsilon}^{\gamma}$ (see Fig. 2) that have a macroscopic shape $\Omega$ and consist of layers of open, straight beams with square cross section of diameter $\sim \varepsilon$. Inside a MBS $\Omega_{\varepsilon}^{\gamma}$ the beams are arranged just like in a cord-belt with cord-angle $0 \leq \gamma \leq \frac{\pi}{2}$. The initial contact zone within the MBSs $\Omega_{\varepsilon}^{\gamma}$, i.e. the union of the beam surfaces inside $\Omega$, is denoted $\Gamma_{C, \varepsilon}^{\gamma}$. All the beams involved in a MBS $\Omega_{\varepsilon}^{\gamma}$ are assumed to be made from a hyperelastic material with a polyconvex elastic energy density $W: \mathrm{M}^{3} \rightarrow[0, \infty]$ showing the correct mechanical behavior under large strain and large compression. Prototypical for our purposes is the elastic energy density 
of a Neo-Hookean material

$$
W(F)= \begin{cases}\alpha_{1}|F|^{p}+\alpha_{2}(\operatorname{det} F)^{\frac{p}{3}}+\alpha_{3} \frac{1}{(\operatorname{det} F)^{\sigma}} & \text { if } \operatorname{det} F>0 \\ \infty & \text { else, }\end{cases}
$$

with $p>3$ and $\alpha_{1}, \alpha_{2}, \alpha_{3}, \sigma$ positive constants. Moreover, the beams are assumed to exhibit an initial brittle bond with their neighbors. We suppose the nature of this bond to be described by a surface energy density $\theta:[0, \infty) \rightarrow[0, \infty)$ whose prototype is a Griffith-like

$$
\theta(t)= \begin{cases}0 & \text { if } t=0 \\ \alpha_{\text {Griffith }}+\theta_{\text {interact }}(t) & \text { else }\end{cases}
$$

for a monotone increasing, concave and continuous $\theta_{\text {interact }}$ with $\theta_{\text {interact }}(0)=0$ and $\alpha_{\text {Griffith }}$ a positive constant. The kinematic constraints we impose on the MBSs $\Omega_{\varepsilon}^{\gamma}$ reflect the needs of large deformation mechanics. That is, deformations $\varphi: \Omega_{\varepsilon}^{\gamma} \rightarrow \mathbb{R}^{3}$ have to preserve the local orientation, i.e. $\operatorname{det} \nabla \varphi>0$, and have to guarantee noninterpenetration of matter, i.e. deformations $\varphi$ have to be injective. To this end we employ the Ciarlet-Nečas condition

$$
\operatorname{vol} \varphi\left(\Omega_{\varepsilon}^{\gamma}\right) \geq \int_{\Omega_{\varepsilon}^{\gamma}}|\operatorname{det} \nabla \varphi| \mathrm{d} x
$$

Indeed, in $[8,15]$ it has been shown that the validity of the Ciarlet-Nečas condition for $\varphi$ together with a.e.positivity of the Jacobian determinant det $\nabla \varphi$ imply injectivity of the deformation $\varphi$ up to a set of zero volume. For technical reasons we impose also a confinement condition, i.e. we ask all deformations of a MBS $\Omega_{\varepsilon}^{\gamma}$ to obtain values in a compact set Box with nonempty interior only. Upon identifying deformations of $\Omega_{\varepsilon}^{\gamma}$ a.e. with deformations of the macroscopic shape $\Omega$, we denote the set of all kinematically admissible deformations $\operatorname{Kin}(\Omega ;$ Box $)$.

A mathematical model for a MBS $\Omega_{\varepsilon}^{\gamma}$ is then given by its total energy

$\mathcal{F}_{\varepsilon}^{\gamma}: \operatorname{SBV}^{p}\left(\Omega ; \mathbb{R}^{3}\right) \cap \operatorname{Kin}(\Omega ; \mathbf{B o x}) \rightarrow[0, \infty]$

$$
\mathcal{F}_{\varepsilon}^{\gamma}(\varphi)= \begin{cases}\int_{\Omega_{\varepsilon}^{\gamma}} W(\nabla \varphi) \mathrm{d} x+\int_{\Gamma_{C, \varepsilon}^{\gamma}} \theta\left(\left|\varphi^{+}-\varphi^{-}\right|\right) \mathrm{d} \mathcal{H}^{2} \\ \infty & \text { if } \varphi \in \mathrm{W}^{1, p}\left(\Omega_{\varepsilon}^{\gamma} ; \mathbb{R}^{3}\right), \\ \text { else. }\end{cases}
$$

Herein, $\operatorname{SBV}^{p}\left(\Omega ; \mathbb{R}^{3}\right)$ is the subspace of the special functions of bounded variation in $\Omega$ whose elements have $p$-integrable approximate differential $\nabla \varphi$ and a discontinuity set $S_{\varphi}$ of finite 2-dimensional Hausdorff-measure $\mathcal{H}^{2}$. The values $\varphi^{ \pm}$denote the traces of $\varphi$ on opposite sides of the discontinuity set $S_{\varphi}$. Our analysis focuses on the asymptotics of $\left(\mathcal{F}_{\varepsilon}^{\gamma}\right)_{\varepsilon}$ as $\varepsilon$, i.e. the beam diameter in $\Omega_{\varepsilon}^{\gamma}$, tends to zero while the cord-angle $\gamma$ remains fixed. To this end we study $\Gamma$-convergence properties of $\left(\mathcal{F}_{\varepsilon}^{\gamma}\right)_{\varepsilon}$ w.r.t. strong $\mathrm{L}^{1}\left(\Omega ; \mathbb{R}^{3}\right)$-convergence (see also [6], wherein a wide variety of homogenization problems defined on $\operatorname{SBV}^{p}\left(\Omega ; \mathbb{R}^{3}\right)$ is analyzed with $\Gamma$-convergence methods, but for quite different energy densities and kinematic constraints). We find that for any $0 \leq \gamma \leq \frac{\pi}{2}$ there holds $\Gamma$-convergence of $\left(\mathcal{F}_{\varepsilon}^{\gamma}\right)_{\varepsilon}$ to a homogenization limit $\mathcal{F}_{\text {Hom }}^{\gamma}$ in at least all physically relevant deformations. That is, in all those $\varphi \in \operatorname{SBV}^{p}\left(\Omega ; \mathbb{R}^{3}\right) \cap \operatorname{Kin}(\Omega$; Box $)$ having a "piecewise" regular discontinuity set we prove

$$
\left(\Gamma-\lim _{\varepsilon \rightarrow 0} \mathcal{F}_{\varepsilon}^{\gamma}\right)(\varphi)=\mathcal{F}_{\text {Hom }}^{\gamma}(\varphi)
$$


However, the homogenized MBSs behave quite differently, depending on whether the cord-angle $\gamma$ is zero or nonzero. In case of zero cord-angle $\gamma=0$ the homogenization limit is

$$
\mathcal{F}_{\text {Hom }}^{0}(\varphi)= \begin{cases}\int_{\Omega} W(\nabla \varphi) \mathrm{d} x+\int_{S_{\varphi}}\left(\left|\nu_{\varphi, 2}\right|+\left|\nu_{\varphi, 3}\right|\right) \theta\left(\left|\varphi^{+}-\varphi^{-}\right|\right) \mathrm{d} \mathcal{H}^{2} \\ \infty & \text { if } \nu_{\varphi, 1}=0 \mathcal{H}^{2} \text {-a.e. on } S_{\varphi}, \\ \text { else, }\end{cases}
$$

where $\nu_{\varphi}=\left(\nu_{\varphi, i}\right)_{i=1,2,3}$ is the normal on the discontinuity set $S_{\varphi}$. Hence, for $\gamma=0$ the set of all attainable deformations enlarges during the homogenization process: the homogenized body may parallel to the beam directions in $\Omega_{\varepsilon}^{0}$ break down in fragments of nearly arbitrary shape, each of which shows the same elastic properties like the beams composing $\Omega_{\varepsilon}^{0}$. But the larger set of attainable deformations comes at the cost of anisotropy in the surface energy. In the case of nonzero cord-angle $0<\gamma \leq \frac{\pi}{2}$ the homogenization limit becomes

$$
\mathcal{F}_{\text {Hom }}^{\gamma}(\varphi):= \begin{cases}\int_{\Omega} W(\nabla \varphi) \mathrm{d} x+\int_{S_{\varphi}} \theta\left(\left|\varphi^{+}-\varphi^{-}\right|\right) \mathrm{d} \mathcal{H}^{2} \\
\infty & \begin{array}{l}
\text { if } \nu_{\varphi, 1}=\nu_{\varphi, 2}=0 \mathcal{H}^{2} \text {-a.e. on } S_{\varphi} \\
\text { else },
\end{array}\end{cases}
$$

and is independent of the cord-angle $\gamma$. In contrast to the case of zero-cord angle the set of attainable deformations now becomes smaller during the homogenization process: only deformations with discontinuity sets parallel to the plane generated by the beam directions in $\Omega_{\varepsilon}^{\gamma}$ can be of finite energy. Cracks vertical to this plane, like they were possible in the MBSs $\Omega_{\varepsilon}^{\gamma}$, can no longer be observed in the homogenization limit. This loss of kinematic freedom is accompanied by a gain of isotropy, again in contrast to the case of zero-cord angle: the homogenization limit does not remember the cord-angle. Moreover, in the homogenization limit the MBSs $\Omega_{\varepsilon}^{\gamma}$ with nonzero cord-angle behave like a laminate of thin plates. That is, like a laminate of mechanically 2D objects, although they are composed of beams, i.e. 1D objects. Thus, our homogenization result provides rigorous evidence that MBSs composed of low-dimensional objects can actually replace such composed of higher dimensional objects, provided they have appropriate geometry and are possibly laminated. Another consequence of the MBSs $\Omega_{\varepsilon}^{\gamma}$ with nonzero cord-angle behaving like laminated plates is that they protect against penetration in the homogenization limit. For zero cord-angle one could still stab a knife in $x_{3}$-direction into or through the homogenized MBS. Whereas in the case of nonzero cord-angle the knife could not even penetrate the homogenized MBS because this would result in a (unattainable) discontinuity set which is nonparallel to the plane generated by the beam directions.

One of the principal difficulties we will encounter in the analysis is to provide approximations for $\varphi \in$ $\operatorname{SBV}^{p}\left(\Omega ; \mathbb{R}^{3}\right) \cap \operatorname{Kin}(\Omega ;$ Box $)$ under the restriction that the approximations again be element of the set $\mathrm{SBV}^{p}\left(\Omega ; \mathbb{R}^{3}\right) \cap \operatorname{Kin}(\Omega ; \mathbf{B o x})$. Plainly speaking: given an injective $\mathrm{SBV}^{p}$-deformation, how to construct an approximation in $\mathrm{SBV}^{p}$ which is injective, too? Answering this question is crucial in order to construct recovery sequences in the $\Gamma$-convergence analysis of the total energies $\left(\mathcal{F}_{\varepsilon}^{\gamma}\right)_{\varepsilon}$. To this end we introduce what we call predeformations: a bijective function $\Phi: \Omega \backslash K \rightarrow \Omega^{\prime}$, where $K$ is empty or a compact subset of $\mathbb{R}^{3}$ with finite $\mathcal{H}^{2}$-mass and $\Omega^{\prime}$ an open subset of $\Omega$, is said to be a predeformation, if:

- $\Phi$ is continuous and of class $\mathrm{W}^{1, \infty}\left(\Omega \backslash K ; \mathbb{R}^{3}\right)$,

- $\Phi^{-1}$ is Lipschitz,

- $\operatorname{det} \mathrm{D} \Phi>0$ a.e.

We prove that composition of deformation and predeformation preserves the property of being a special function of bounded variation and also the kinematic constraints. More precisely

$$
\varphi \in \operatorname{SBV}^{p}\left(\Omega ; \mathbb{R}^{3}\right) \cap \operatorname{Kin}(\Omega ; \mathbf{B o x}) \quad \Rightarrow \quad \varphi \circ \Phi \in \operatorname{SBV}^{p}\left(\Omega ; \mathbb{R}^{3}\right) \cap \operatorname{Kin}(\Omega ; \operatorname{Box})
$$


and moreover

$$
S_{\varphi \circ \Phi} \subseteq \Phi^{-1}\left(S_{\varphi} \cap \Omega^{\prime}\right) \cup K
$$

The last inclusion reveals that the discontinuity set of the composition deformation-predeformation is contained in the union of the inverse image of the deformation's discontinuity set under the predeformation and a "seam" of Hausdorff-dimension 2. This makes predeformations a suitable tool to manipulate a deformation's discontinuity set and to provide approximations inside $\operatorname{SBV}^{p}\left(\Omega ; \mathbb{R}^{3}\right) \cap \operatorname{Kin}(\Omega ; \mathbf{B o x})$.

\section{Outline of the article}

In Section 2 we fix the basic notation. Section 3 is entirely devoted to the modeling of the MBSs $\Omega_{\varepsilon}^{\gamma}$. Furthermore, at the end of Section 3 we include the Euler-Lagrange equations of the corresponding total energies $\mathcal{F}_{\varepsilon}^{\gamma}$, together with a discussion of the mechanical reality that the mathematical model actually describes. Section 4 contains the mathematical tools we need in the analysis including the concept of predeformations. Our homogenization results are stated and proved in the final Section 5.

\section{BASIC DEFINITIONS AND NOTATION}

In this section we provide the reader with the basic definitions and notation used throughout the article.

\section{Domains, balls, spheres}

Let $(V,\|\cdot\|)$ be a real normed vector space, $M$ a subset of $V, \lambda \in \mathbb{R}$ and $b \in V$. We call the subset $M$ a domain, if it is open and connected. The boundary of $M$ is denoted by $\partial M$, its $\lambda$-homothety, i.e. $\{\lambda v: v \in M\}$ by $\lambda M$ and the translation of $M$ by the vector $b$, i.e. $\{b+v: v \in M\}$, is denoted $b+M$. The open ball with radius $r>0$ around $x \in V$ is $B_{r}(x)$. In case $V$ is $\mathbb{R}^{N}, N \in \mathbb{N}$, equipped with the Euclidean norm we refer to the unit sphere as $S^{N-1}$. Finally, the set indicator function $\mathbb{1}_{M}$ of $M$ is defined through $\mathbb{1}_{M}(v)=1$ for $v \in M$ and 0 elsewhere in $V$.

\section{Vectors and matrices}

The components of a vector $u \in \mathbb{R}^{N}$ are referred to as $u_{i}, i \in\{1, \ldots, N\}$. In case a vector already carries an index, e.g. $u_{\text {index }}$, we denote its $i$ th component by $u_{\text {index }, i}$. When we write $u$ by means of its components, we either use the notation $u=\left(u_{1}, \ldots, u_{N}\right)$, or $u=\left[u_{1}, \ldots, u_{N}\right]^{T}$ in order to emphasize its nature as a column vector. By $e_{i}$ we denote the $i$ th unit vector in $\mathbb{R}^{N}$, that is $e_{i, i}=1$ and $e_{i, j}=0$ for $j \neq i$. Furthermore we suppose $\mathbb{R}^{N} \ni u, v$ to be endowed with the standard scalar product $u \cdot v:=\sum_{i=1}^{N} u_{i} v_{i}$ inducing the Euclidean $\operatorname{norm}|u|:=\sqrt{u \cdot u}$.

The set of all real $N \times N$-matrices is denoted $\mathrm{M}^{N}$. We refer to the components of a matrix $F \in \mathrm{M}^{N}$ as $F_{i j}, i, j \in\{1, \ldots, N\}$. By means of its components $F$ is written as $\left[F_{i j}\right]_{i, j=1, \ldots, N}$. The standard scalar product in $\mathbb{M}^{N} \ni F, M$ is denoted $F: M=\sum_{i, j=1}^{N} F_{i j} M_{i j}$ inducing the Euclidean norm $|F|:=\sqrt{F: F}$. For two vectors $u, v \in \mathbb{R}^{N}$ the quantity $u \otimes v:=\left[u_{i} v_{j}\right]_{i, j=1, \ldots, N} \in \mathbb{M}^{N}$ defines the outer product of $u$ and $v$. The identity matrix in $\mathbb{M}^{N}$ is denoted by $I$. The determinant of $F \in \mathbb{M}^{N}$ is $\operatorname{det} F$ and for invertible $F$ the quantity Cof $F:=(\operatorname{det} F) F^{-T}$ is called the cofactor matrix of $F$.

We define $\mathrm{M}_{>}^{N}$ to be the set of all real $N \times N$ matrices with positive determinant, and $\operatorname{SO}(N)$ to be the set of all rotations, i.e. the set of all $Q \in \mathrm{M}^{N}$ such that $Q^{T} Q=I$ and $\operatorname{det} Q=1$.

\section{Measure theoretical terms}

The Lebesgue measure in $\mathbb{R}^{k}$ is referred to as $\operatorname{vol}_{k}$, and in $\mathbb{R}^{N}$ simply as vol. Whereas $\mathcal{H}^{N-1}$ denotes the $(N-1)$-dimensional Hausdorff-measure (in $\left.\mathbb{R}^{N}\right)$. 
Let $\Omega$ be an open and bounded subset of $\mathbb{R}^{N}$. For $\varphi \in \mathrm{L}^{1}\left(\Omega ; \mathbb{R}^{N}\right)$ a point $x_{0} \in \Omega$ is said to be a point of approximate continuity of $\varphi$, if there is a $z \in \mathbb{R}^{N}$ such that

$$
\lim _{r \rightarrow 0} \frac{1}{\operatorname{vol} B_{r}\left(x_{0}\right)} \int_{B_{r}\left(x_{0}\right)}|\varphi(x)-z| \mathrm{d} x=0 .
$$

In this case $z$ is called the approximate limit of $\varphi$ in $x_{0}$, in symbols $z=\operatorname{aplim}_{y \rightarrow x_{0}} \varphi(y)$. The Borel set of all points in which $\varphi$ is not approximately continuous is denoted $S_{\varphi}$ and referred to as the (approximate) discontinuity set of $\varphi$. Moreover, we say that $\varphi$ is approximately differentiable in $x_{0} \in \Omega$, if it is approximately continuous in $x_{0}$ and there is an $M \in \mathbb{I}^{N}$ satisfying

$$
\lim _{r \rightarrow 0} \frac{1}{\operatorname{vol} B_{r}\left(x_{0}\right)} \int_{B_{r}\left(x_{0}\right)} \frac{\left|\varphi(x)-\operatorname{aplim}_{y \rightarrow x_{0}} \varphi(y)-M\left(x-x_{0}\right)\right|}{r} \mathrm{~d} x=0 .
$$

In this case $M$ is called the approximate differential of $\varphi$ in $x_{0}$ and is denoted ap $\mathrm{D} \varphi\left(x_{0}\right)$. The set of all points in which $\varphi$ is approximately differentiable is written $\Omega_{\varphi, \mathrm{D}}$.

\section{Polyhedral sets and piecewise $\mathbf{C}^{\mathbf{1}}$-hypersurfaces}

For $k \leq N$, a $k$-dimensional simplex in $\mathbb{R}^{N}$ is the convex hull of $k+1$ points that are not contained in a $(k-1)$-dimensional hyperplane of $\mathbb{R}^{N}$. A subset $P$ of $\mathbb{R}^{N}$ is called polyhedral, if it can be written as the union of finitely many $(N-1)$-dimensional simpleces, called the faces of $P$. The set of all faces of $P$ is denoted Face $(P)$. We assume that two different faces of a polyhedral set in $\mathbb{R}^{N}$ share at maximum a set of dimension $N-2$. In three space dimensions $N=3$ the faces of a polyhedral set are triangles, the faces of a polyhedral set in two space dimensions $N=2$ are lines. For a polyhedral set $P$ in $\mathbb{R}^{2}$ we moreover call $\operatorname{Knot}(P)$ the finite set containing the knots and endpoints of $P$.

A closed subset $S$ of $\mathbb{R}^{N}$ is said to be a piecewise $\mathrm{C}^{1}$-hypersurface of simply piecewise $\mathrm{C}^{1}$, if there are finitely many bounded Lipschitzian domains $U_{1}, \ldots, U_{k} \subseteq \mathbb{R}^{N-1}, g_{i} \in \mathrm{C}^{1}\left(\overline{U_{i}}\right), Q_{i} \in \operatorname{SO}(N)$ and $b_{i} \in \mathbb{R}^{N}$ such that upon setting

there hold:

$$
S_{i}:=\left\{Q_{i}\left[\hat{\xi}, g_{i}(\hat{\xi})\right]^{T}+b_{i}: \hat{\xi} \in U_{i}\right\}
$$

(i) $S=\bigcup_{i=1}^{k} \overline{S_{i}}$

(ii) $S_{i} \cap S_{j}=\emptyset \quad$ for $i \neq j$,

(iii) $\mathbb{R}^{N} \backslash S$ satisfies the cone condition [1], Definition 4.6.

Remark 2.1. Every polyhedral set is also piecewise $\mathrm{C}^{1}$. Moreover, for every piecewise $\mathrm{C}^{1}$-subset $S$ of $\mathbb{R}^{N}$ a unit normal $\nu_{S}$ exists $\mathcal{H}^{N-1}$-a.e. (and is determined up to its sign). Finally, given $S$ as the union of $\overline{S_{1}}, \ldots, \overline{S_{k}}$ like above, it is $\mathcal{H}^{N-1}\left(\overline{S_{i}} \cap \overline{S_{j}}\right)=0$ for $i \neq j$.

For a domain $\Omega$ such that $\mathbb{R}^{N} \backslash \partial \Omega$ satisfies the cone condition we say that a subset $S$ of $\bar{\Omega}$ is piecewise $\mathrm{C}^{1}$ in $\Omega$, if $S$ is a piecewise $\mathrm{C}^{1}$-hypersurface and $\mathbb{R}^{N} \backslash(\partial \Omega \cup S)$ satisfies the cone condition.

\section{Regularity of domains}

A domain $\Omega$ in $\mathbb{R}^{N}$ is called Lipschitzian, if it has a Lipschitz boundary in the sense of [13], Section 4.2. Furthermore, we call a domain $\Omega$ nonoscillating, if the intersection of $\Omega$ with an arbitrary $(N-1)$-dimensional simplex has a finite number of connected components. Thus, the intersection of a two-dimensional nonoscillating domain with a line segment is a finite union of line segments. 
Remark 2.2 (on nonoscillating domains). The property of being nonoscillating is not connected to the smoothness of a domain's boundary. A counterexample in two-space dimensions is a $\mathrm{C}^{\infty}$-domain whose boundary is in a neighborhood of 0 given by the graph of the $\mathrm{C}^{\infty}$-function $x \mapsto \mathbb{1}_{(0, \infty)}(x) \mathrm{e}^{-1 / x} \sin (1 / x)$.

\section{Lebesgue- and Sobolev-spaces}

For $\Omega$ an open and bounded subset of $\mathbb{R}^{N}$, the Lebesgue-spaces $\mathrm{L}^{p}\left(\Omega ; \mathbb{R}^{M \times K}\right)$ and the Sobolev-spaces $\mathrm{W}^{1, p}\left(\Omega ; \mathbb{R}^{M}\right), 1 \leq p \leq \infty$, are assumed to be defined in the standard sense. Their respective norms are denoted by $\|\cdot\|_{\mathrm{L}^{p}\left(\Omega ; \mathbb{R}^{M \times K}\right)}$ and $\|\cdot\|_{\mathrm{W}^{1, p}\left(\Omega ; \mathbb{R}^{M}\right)}$.

\section{Mathematical Model}

We now expose the mathematical model for the cord-belt like MBSs motivated previously in the introduction. It is entirely formulated in terms of geometrically and constitutively nonlinear solid mechanics. At the end of the section we state the Euler-Lagrange equations for the total energies associated with the MBSs and give an interpretation of the mechanical reality that our model actually describes.

\section{Geometry}

The microstructures $\mathcal{D}_{\gamma}$ we introduce here are to resemble the geometry of cord-belts like they were sketched in the introduction. For cord-angles $0 \leq \gamma \leq \frac{\pi}{2}$ we define

$$
\mathcal{D}_{\gamma}:=\bigcup\left\{\left(0, a_{2}, 2 a_{3}\right)+\mathcal{B}: a_{2}, a_{3} \in \mathbb{Z}\right\} \cup R_{\gamma}\left(\bigcup\left\{\left(0, a_{2}, 2 a_{3}-1\right)+\mathcal{B}: a_{2}, a_{3} \in \mathbb{Z}\right\}\right)
$$

Therein, $\mathcal{B}:=\mathbb{R} \times(0,1)^{2}$ is an infinitely long beam with square cross section and $R_{\gamma}$ is the rotation about the axis of the third component through $\gamma$. Like cord-belts the microstructures $\mathcal{D}_{\gamma}$ are composed of layers of slender objects which are oriented coherently inside one layer while the directions alternate in adjacent layers by the cord-angle $\gamma$. The cord-belt like MBSs $\Omega_{\varepsilon}^{\gamma}$ we study in this article (see Fig. 2) are now obtained by scaling down the microstructures $\mathcal{D}_{\gamma}$ by a small positive parameter $\varepsilon$ and intersecting the homothety with a domain $\Omega$. We advice the reader that $\varepsilon$ quantifies the beam diameter in the resulting MBSs $\Omega_{\varepsilon}^{\gamma}$, while $\Omega$ is the outer, macroscopic shape of $\Omega_{\varepsilon}^{\gamma}$.

Definition of Geometry 3.1 (MBS with zero cord-angle). Let $\omega \subseteq \mathbb{R}^{2}$ be a bounded and nonoscillating Lipschitzian domain, $\ell>0$ and $\Omega:=(0, \ell) \times \omega$ be a beam-like cylinder. The MBS $\Omega_{\varepsilon}^{0}$ with macroscopic shape $\Omega$ and zero cord-angle $\gamma=0$ and the corresponding inner contact boundary $\Gamma_{C, \varepsilon}^{0}$ are respectively defined as

$$
\Omega_{\varepsilon}^{0}:=\Omega \cap \varepsilon \mathcal{D}_{0} \quad \text { and } \quad \Gamma_{C, \varepsilon}^{0}:=\Omega \cap \partial \Omega_{\varepsilon}^{0} .
$$

Definition of Geometry 3.2 (MBS with nonzero cord-angle). Let $\omega \subseteq \mathbb{R}^{2}$ be a bounded Lipschitzian domain, $a>0$ and $\Omega:=\omega \times(-a, a)$ be a plate-like cylinder. The MBS $\Omega_{\varepsilon}^{\gamma}$ with macroscopic shape $\Omega$ and nonzero cordangle $0<\gamma \leq \frac{\pi}{2}$ and the corresponding inner contact boundary $\Gamma_{C, \varepsilon}^{\gamma}$ are respectively defined as

$$
\Omega_{\varepsilon}^{\gamma}:=\Omega \cap \varepsilon \mathcal{D}_{\gamma} \quad \text { and } \quad \Gamma_{C, \varepsilon}^{\gamma}:=\Omega \cap \partial \Omega_{\varepsilon}^{\gamma} \text {. }
$$

Remark 3.1. It is due to technical reasons that we restrict the macroscopic shapes to beam-like cylinders in Definition of Geometry 3.1 and to plate-like cylinders in Definition of Geometry 3.2. However, these cylinder-like macroscopic shapes are quite natural for the respective microstructures. 


\section{Constitutive relations}

We assume that the beams composing the MBSs $\Omega_{\varepsilon}^{\gamma}$ are made from a homogeneous hyperelastic material with energy density $W$. The energy density $W$ shall have realistic behavior under large strain, large compression and local self-interpenetration: $W(F) \rightarrow \infty$ as $|F| \rightarrow \infty$ or $\operatorname{det} F \searrow 0$ and $W(F)=\infty$ for $\operatorname{det} F \leq 0$. To this end, we rely on the notion of polyconvexity and make the following assumptions on the elastic energy density $W: \mathrm{M}^{3} \rightarrow[0, \infty]:$

$$
\left\{\begin{array}{l}
\text { There is a convex function } \mathbb{W}: \mathbb{M}^{3} \times(0, \infty) \rightarrow[0, \infty) \text { such that } \\
W(F)= \begin{cases}\mathbb{W}(F, \operatorname{det} F) & \text { if } \operatorname{det} F>0, \\
\infty & \text { else, }\end{cases} \\
\text { holds for all } F \in \mathbb{M}^{3} .
\end{array}\right.
$$

Moreover, the function $\mathrm{W}$ shall obey for all $F, M \in \mathrm{M}_{>}^{3}$ the growth conditions

$$
\begin{aligned}
& \mathrm{W}(F, \operatorname{det} F) \geq \alpha_{1}|F|^{p}-\alpha_{2} \quad \text { for } p>3 \text { and positive constants } \alpha_{1}, \alpha_{2}, \\
& \mathbb{W}(F, \operatorname{det} F) \rightarrow \infty \quad \text { as } \operatorname{det} F \searrow 0, \\
& \mathbf{W}(F \cdot M, \operatorname{det}(F \cdot M)) \leq c_{\mathbb{W}}(M) \cdot(\mathbb{W}(F, \operatorname{det} F)+1) \quad \text { for some function } c_{\mathbb{W}} \in C\left(\mathbf{M}_{>}^{3}\right) .
\end{aligned}
$$

We assume the inner contact boundary $\Gamma_{C, \varepsilon}^{\gamma}$ of a MBS $\Omega_{\varepsilon}^{\gamma}$ to be covered with an infinitesimally thin layer of homogeneous adhesive material that forms an initial bond between neighboring beams in $\Omega_{\varepsilon}^{\gamma}$. Brittleness is understood in the sense of Griffith: breaking the bond between two laminated objects comes at a constant energetic cost per debonded unit area. The energy stored in a broken bond per unit area shall be described by a surface energy density $\theta:[0, \infty) \rightarrow[0, \infty)$ which satisfies the following assumptions on the surface energy density. First, the surface energy density shall depend only on the distance of the two surface elements involved in the bond (a consequence of the principle of frame-indifference, see [24], Prop. 2.7). It shall also satisfy some minor regularity:

$$
\theta \text { is lower semicontinuous. }
$$

Moreover, the energy stored in a broken bond shall increase with the distance between the two surface elements involved, in a way that the interaction between them decreases as the distance grows:

$$
\theta \text { is monotone increasing and concave. }
$$

Reflecting the constant energetic cost for debonding per unit area, the surface energy density $\theta$ has to jump in 0 , i.e. from zero distance between the two surface elements involved to nonzero distance

$$
\theta(0)=0 \text { and } \lim _{t \searrow 0} \theta(t)=\alpha_{\text {Griffith }} \quad \text { for a positive constant } \alpha_{\text {Griffith }}
$$

\section{Kinematics}

Any reasonable model for MBSs has to guarantee that the subbodies involved do not interpenetrate when they come into mechanical contact. The fact that mechanical contact is a phenomenon that occurs in the deformed configuration makes it particularly difficult to deal with in the large deformation regime.

Noninterpenetration in its very sense means that two different mass points may never occupy the same spatial position. In other words, a deformation $\varphi$ of an open $N$-dimensional "body" $U$ (not necessarily connected) guarantees noninterpenetration of matter, if and only if it is injective. Ciarlet and Nečas [8] observed that for an injective deformation the volume of the deformed body cannot be less than the volume the mass points occupy in the deformed configuration. This alternative statement of noninterpenetration of matter, called the Ciarlet-Nečas condition, proves much handier than demanding injectivity, especially for only a.e. defined deformations. But it requires a notion for the image of a body under only a.e. defined deformations. Recently, 
this notion was introduced by Giacomini and Ponsiglione [15]: let $U$ be an open and bounded subset of $\mathbb{R}^{N}$ and $\varphi \in \mathrm{L}^{1}\left(U ; \mathbb{R}^{N}\right)$ be a.e. approximately differentiable. The measure theoretical image (or deformed configuration) of an arbitrary subset $E \subseteq U$ under $\varphi$, denoted $[\varphi(E)]$, is defined as

$$
[\varphi(E)]:=\left\{\operatorname{ap}_{y \rightarrow x} \varphi(y): x \in E \cap U_{\varphi, \mathrm{D}}\right\} \cup\left\{0: x \in E \backslash U_{\varphi, \mathrm{D}}\right\}
$$

Recall that $U_{\varphi, \mathrm{D}}$ is the set of all points of approximate differentiability of $\varphi$. Now the Ciarlet-Nečas condition for an a.e. approximately differentiable $\varphi \in \mathrm{L}^{1}\left(U ; \mathbb{R}^{N}\right)$ reads

$$
\operatorname{vol}[\varphi(U)] \geq \int_{U}|\operatorname{det}(\operatorname{ap} \mathrm{D} \varphi)| \mathrm{d} x
$$

Remark 3.2. A deformation $\varphi$ satisfies the Ciarlet-Nečas condition, if and only if for all measurable subsets $E \subseteq U$ inequality (3.1) is satisfied with $E$ replacing $U$. Furthermore, the Ciarlet-Nečas condition for a deformation $\varphi$ is equivalent to demanding equality in (3.1). In other words, a deformation satisfies the Ciarlet-Nečas condition, if and only if the volume of the deformed body equals the volume the material occupies in the deformed configuration. See [24], Remark 2.10, for details.

Generalizing results of Ciarlet and Nečas [8], Giacomini and Ponsiglione revealed in [15], Proposition 2.5, that the Ciarlet-Nečas condition is an equivalent statement for injectivity.

Proposition 3.3. Let $U$ be an open and bounded subset of $\mathbb{R}^{N}, \varphi \in \mathrm{L}^{1}\left(U ; \mathbb{R}^{N}\right)$ be approximately differentiable a.e. and assume $\operatorname{det}(\operatorname{ap} \mathrm{D} \varphi) \neq 0$ a.e. Then $\varphi$ satisfies the Ciarlet-Nečas condition, if and only if $\varphi$ is a.e.injective in $U$, i.e. if and only if there is a representative $\bar{\varphi}$ of $\varphi$ and a measurable subset $M$ of $U$ with $\operatorname{vol} M=0$ such that $\bar{\varphi}$ is injective on $U \backslash M$.

Remark 3.3. The main flaw of the Ciarlet-Nečas condition as a statement of noninterpenetration of matter is that it ensures injectivity only up to a set of zero volume. To put it simply, the Ciarlet-Nečas condition guarantees nonoverlapping in the deformed configuration but is still open to (self-)intersections. In some cases this may lead to deformed configurations that are compatible with the Ciarlet-Nečas condition but cannot be realized without previous interpenetration, see [20], Figure 4. Also, the Ciarlet-Nečas condition does not take into account the geometry of the reference configuration. For example, interchanging the positions of two congruent bodies is clearly allowed by the Ciarlet-Nečas condition, although sometimes the reference configuration suggests that such interchange is unlikely. A recent progress in the mathematical treatment of noninterpenetration of matter is [20].

In addition to the Ciarlet-Nečas condition, we demand deformations of a MBS $\Omega_{\varepsilon}^{\gamma}$ to satisfy a confinement condition: a.a. mass points of $\Omega_{\varepsilon}^{\gamma}$ must be moved into a compact subset Box of $\mathbb{R}^{3}$ with nonempty interior which we furthermore assume to be rigid.

Remark 3.4. In many applications the deformation of a solid body is not determined by the position of the body's boundary but by a rigid environment. Such-like situations are mostly encountered when the body under consideration is "small" and "soft" compared to its environment. The rigid environment can be modeled by imposing a confinement condition like Box.

Finally, all deformations of a MBS $\Omega_{\varepsilon}^{\gamma}$ shall preserve the local orientation, i.e. are supposed to have an a.e. positive Jacobian determinant.

The kinematic constraints we impose on deformations of a MBS $\Omega_{\varepsilon}^{\gamma}$ are the following. A deformation $\varphi \in \mathrm{L}^{1}\left(\Omega_{\varepsilon}^{\gamma} ; \mathbb{R}^{3}\right)$ - which can be identified with an element of $\mathrm{L}^{1}\left(\Omega ; \mathbb{R}^{3}\right)$ - is kinematically admissible, if it is 
an element of the set

$$
\begin{aligned}
& \operatorname{Kin}(\Omega ; \operatorname{Box}):=\left\{\varphi: \varphi \in \mathrm{L}^{1}\left(\Omega ; \mathbb{R}^{3}\right) \text { such that for a.e. } x \in \Omega\right. \text { it is } \\
& \varphi \text { approximately differentiable in } x, \operatorname{det}(\operatorname{ap} \mathrm{D} \varphi(x))>0 \text {, } \\
& \left.\varphi(x) \in \mathbf{B o x}, \text { and } \operatorname{vol}[\varphi(\Omega)] \geq \int_{\Omega}|\operatorname{det}(\operatorname{apD} \varphi)| \mathrm{d} x\right\} \text {. }
\end{aligned}
$$

The set $\operatorname{Kin}(\Omega ; \mathbf{B o x})$ is indeed nonempty regarding the nonempty interior of Box. By Proposition 3.3 it is the set of all the a.e.-injective $\mathrm{L}^{1}\left(\Omega ; \mathbb{R}^{3}\right)$-deformations that preserve the local orientation and take a.a. values in Box.

\section{External loads}

The MBSs $\Omega_{\varepsilon}^{\gamma}$ shall be exposed to a conservative applied (follower-) body load given by a potential $F$ that only depends on the mass point and its spatial position in the deformed configuration. We impose the following assumptions on the potential of the (follower-) body load $F: \Omega \times$ Box $\rightarrow \mathbb{R}$ :

$$
\begin{aligned}
& \left\{\begin{array}{l}
\text { The mapping } \\
\left\{\varphi: \varphi \in \mathrm{L}^{1}\left(\Omega ; \mathbb{R}^{3}\right), \varphi(x) \in \text { Box a.e. }\right\} \ni \varphi \mapsto \int_{\Omega} F(x, \varphi(x)) \mathrm{d} x \\
\text { is well-defined and continuous w.r.t. strong } \mathrm{L}^{1}\left(\Omega ; \mathbb{R}^{3}\right) \text {-convergence. }
\end{array}\right. \\
& |F(x, v)| \leq \alpha_{\text {force }} \text { for all } x \in \Omega, v \in \text { Box and a positive constant } \alpha_{\text {force }}
\end{aligned}
$$

A nontrivial example for such a (follower-) body load potential are centrifugal forces [24], Section 2.2.4.

\section{Total energy and Euler-Lagrange equations}

Given the elasticity hypothesis on the beams composing a MBS $\Omega_{\varepsilon}^{\gamma}$, the constitutive assumptions $(W 1), \ldots$, $(W 3)$ and $(\theta 1), \ldots,(\theta 3)$, the kinematic constraints $\operatorname{Kin}(\Omega ; \mathbf{B o x})$ and the assumptions on the body load potential $(F 1),(F 2)$ we can define the total energy associated with a MBS $\Omega_{\varepsilon}^{\gamma}$ through

$$
\begin{aligned}
& \mathcal{E}_{\varepsilon}^{\gamma}: \mathrm{W}^{1, p}\left(\Omega_{\varepsilon}^{\gamma} ; \mathbb{R}^{3}\right) \cap \operatorname{Kin}(\Omega ; \text { Box }) \rightarrow(-\infty, \infty] \\
& \mathcal{E}_{\varepsilon}^{\gamma}(\varphi):=\int_{\Omega_{\varepsilon}^{\gamma}} W(\mathrm{D} \varphi) \mathrm{d} x+\int_{\Gamma_{C, \varepsilon}^{\gamma}} \theta\left(\left|\varphi^{+}-\varphi^{-}\right|\right) \mathrm{d} \mathcal{H}^{2}-\int_{\Omega_{\varepsilon}^{\gamma}} F(x, \varphi(x)) \mathrm{d} x .
\end{aligned}
$$

Theorem 3.4 (existence of minimizers for the total energy $\left.\mathcal{E}_{\varepsilon}^{\gamma}\right)$. Let $\Omega_{\varepsilon}^{\gamma}$ be one of the MBSs given in Definition of Geometry 3.1 or 3.2. Assume that the elastic energy density $W$ satisfies $(W 1), \ldots,(W 3)$, the surface energy density $\theta$ the conditions $(\theta 1), \ldots,(\theta 3)$ and the potential $F$ of the body load $(F 1),(F 2)$. Then the total energy $\mathcal{E}_{\varepsilon}^{\gamma}$ has a minimizer in $\mathrm{W}^{1, p}\left(\Omega_{\varepsilon}^{\gamma} ; \mathbb{R}^{3}\right) \cap \operatorname{Kin}(\Omega ; \mathbf{B o x})$.

The result is proved in Section 5. We conclude the modeling of the MBSs $\Omega_{\varepsilon}^{\gamma}$ with a statement of the formal Euler-Lagrange equations for the associated total energy $\mathcal{E}_{\varepsilon}^{\gamma}$. Since we work in the context of large deformations, we have to transform the Euler-Lagrange equations into the deformed configuration. Only this way we may interpret them as equilibrium equations in the sense of static mechanics. Let us assume that the energy densities $W(F), \theta(t)$ and $F(x, v), F \in \mathbb{M}_{>}^{3}, t>0$ and $v \in \mathbf{B o x}$, and the boundaries of Box and $\Omega$ are sufficiently regular. The first Piola-Kirchhoff stress is denoted $T(F)=\mathrm{D} W(F)$. By $f_{\text {back }}(t)=\frac{\mathrm{d} \theta}{\mathrm{d} t}(t)$ we denote the backdriving force generated by the surface energy density $\theta$ and the applied body load per undeformed unit volume is $f(x, v)=\frac{\partial F}{\partial v}(x, v)$. Let $n: \partial \Omega \cup \Gamma_{C, \varepsilon}^{\gamma} \rightarrow S^{2}$ be a normal field that coincides with the outer normal on $\partial \Omega$. For this normal field we adopt the following convention: given a sufficiently regular function $h: \Omega_{\varepsilon}^{\gamma} \rightarrow \mathbb{R}^{M}$ and 
$x \in \Gamma_{C, \varepsilon}^{\gamma}$, then $h^{ \pm}(x)$ is the $\operatorname{limit}_{\lim _{n}} h\left(x_{n}\right)$ where the $x_{n} \in \Omega_{\varepsilon}^{\gamma}$ are taken from the side of $\Gamma_{C, \varepsilon}^{\gamma}$ which $\pm n(x)$ points to and converge to $x$.

Consider a minimizer $\varphi \in \mathrm{W}^{1, p}\left(\Omega_{\varepsilon}^{\gamma} ; \mathbb{R}^{3}\right) \cap \operatorname{Kin}(\Omega ;$ Box $)$ of the total energy $\mathcal{E}_{\varepsilon}^{\gamma}$ and suppose it is smooth enough. Adopting the notation of Ciarlet [7], Chapters 1 and 2, we refer to elements of the deformed MBS $\varphi\left(\Omega_{\varepsilon}^{\gamma}\right)$ as follows. If $U$ is the reference configuration $\Omega_{\varepsilon}^{\gamma}$ or its outer boundary $\partial \Omega \cap \partial \Omega_{\varepsilon}^{\gamma}$ and $x \in U$, then $x^{\varphi} \in \varphi(U)$ denotes the point $x^{\varphi}=\varphi(x)$. In the case $x^{\varphi} \in \varphi\left(\partial \Omega \cap \partial \Omega_{\varepsilon}^{\gamma}\right)$ the term $\mathrm{d} a^{\varphi}\left(x^{\varphi}\right)$ denotes the area element on $\varphi\left(\partial \Omega \cap \partial \Omega_{\varepsilon}^{\gamma}\right)$ around $x^{\varphi}$. According to [7], Theorem 1.7-1, it is

$$
\mathrm{d} a^{\varphi}\left(x^{\varphi}\right)=|\operatorname{Cof} \mathrm{D} \varphi(x) n(x)| \mathrm{d} a(x),
$$

$\mathrm{d} a(x)$ being the area element on $\partial \Omega \cap \partial \Omega_{\varepsilon}^{\gamma}$ around $x$. For $x \in \Gamma_{C, \varepsilon}^{\gamma}$ we define $x^{\varphi,+}=\varphi^{+}(x) \in \varphi^{+}\left(\Gamma_{C, \varepsilon}^{\gamma}\right)$, the deformed normal on $\varphi^{+}\left(\Gamma_{C, \varepsilon}^{\gamma}\right)$ in $x^{\varphi,+}$ being $n^{\varphi,+}\left(x^{\varphi,+}\right)$. We write $\mathrm{d} a^{\varphi,+}\left(x^{\varphi,+}\right)$ for the area element on $\varphi^{+}\left(\Gamma_{C, \varepsilon}^{\gamma}\right)$ around $x^{\varphi,+}$ which is related to $\mathrm{d} a(x)$ like $(3.2)$. In the same way we understand $x^{\varphi,-} \in \varphi^{-}\left(\Gamma_{C, \varepsilon}^{\gamma}\right)$, $n^{\varphi,-}\left(x^{\varphi,-}\right)$ and $\mathrm{d} a^{\varphi,-}\left(x^{\varphi,-}\right)$. For $x^{\varphi} \in \varphi\left(\Omega_{\varepsilon}^{\gamma}\right)$ and $M: \varphi\left(\Omega_{\varepsilon}^{\gamma}\right) \rightarrow \mathbb{M}^{3}$ a smooth matrix-valued function the quantity $\operatorname{div}^{\varphi} M\left(x^{\varphi}\right)$ denotes the usual divergence of $M$ in the point $x^{\varphi}$. Finally, the Cauchy-stress $T^{\varphi}\left(x^{\varphi}\right)$ in $x^{\varphi}=\varphi(x) \in \varphi\left(\Omega_{\varepsilon}^{\gamma}\right)$ and the first Piola-Kirchhoff stress $T(\mathrm{D} \varphi(x))$ are related by the Piola-transformation

$$
T^{\varphi}\left(x^{\varphi}\right)=T(\mathrm{D} \varphi(x))(\operatorname{Cof} \mathrm{D} \varphi(x))^{-1} .
$$

The applied body load $f^{\varphi}\left(x^{\varphi}\right)$ in the deformed configuration $\varphi\left(\Omega_{\varepsilon}^{\gamma}\right)$ is

$$
f^{\varphi}\left(x^{\varphi}\right)=\frac{1}{\operatorname{det} \mathrm{D} \varphi(x)} f(x, \varphi(x)) .
$$

Then the minimizer $\varphi$ of $\mathcal{E}_{\varepsilon}^{\gamma}$ formally solves the following boundary value problem:

1. Confinement. We have

$$
\varphi\left(\Omega_{\varepsilon}^{\gamma}\right) \subseteq \text { Box. }
$$

2. Equilibrium in the deformed configuration. For all $x^{\varphi} \in \varphi\left(\Omega_{\varepsilon}^{\gamma}\right)$ there holds

$$
-\operatorname{div}^{\varphi} T^{\varphi}\left(x^{\varphi}\right)=f^{\varphi}\left(x^{\varphi}\right) .
$$

3. Conditions on the deformed outer boundary. For all $x^{\varphi} \in \varphi\left(\partial \Omega \cap \partial \Omega_{\varepsilon}^{\gamma}\right)$ we have

$$
T^{\varphi}\left(x^{\varphi}\right) n^{\varphi}(x)=\lambda^{\varphi}\left(x^{\varphi}\right) n^{\varphi}\left(x^{\varphi}\right)
$$

wherein the real number $\lambda^{\varphi}\left(x^{\varphi}\right)$ corresponds to:

(i) $x^{\varphi} \in$ int Box and $\varphi^{-1}\left(\left\{x^{\varphi}\right\}\right)=\{x\}$, then $\lambda^{\varphi}\left(x^{\varphi}\right)=0$,

(ii) $x^{\varphi} \in \partial \mathbf{B o x}$, then $\varphi^{-1}\left(\left\{x^{\varphi}\right\}\right)=\{x\}$ and $\lambda^{\varphi}\left(x^{\varphi}\right) \leq 0$,

(iii) $x^{\varphi} \in \operatorname{int}$ Box and $\varphi^{-1}\left(\left\{x^{\varphi}\right\}\right)=\{x, y\}$ for some $y \in \partial \Omega_{\varepsilon}^{\gamma}$, then $\lambda^{\varphi}\left(x^{\varphi}\right) \leq 0$.

4. Conditions on the deformed inner contact boundary. For all $x^{\varphi, \pm} \in \varphi^{ \pm}\left(\Gamma_{C, \varepsilon}^{\gamma}\right)$ we distinguish the following situations.

If there is $r>0$ such that $z^{\varphi,+}=z^{\varphi,-}$ for all $z \in B_{r}(x) \cap \Gamma_{C, \varepsilon}^{\gamma}$, then

$$
-\operatorname{div}^{\varphi} T^{\varphi}\left(x^{\varphi}\right)=f^{\varphi}\left(x^{\varphi}\right) .
$$

If $x^{\varphi,+} \neq x^{\varphi,-}$, then in $x^{\varphi,+}$ there holds

$$
\begin{aligned}
T^{\varphi,+}\left(x^{\varphi,+}\right) & \left(-n^{\varphi,+}\left(x^{\varphi,+}\right)\right) \\
& +f_{\text {back }}\left(\left|x^{\varphi,+}-x^{\varphi,-}\right|\right) \frac{x^{\varphi,+}-x^{\varphi,-}}{\left|x^{\varphi,+}-x^{\varphi,-}\right|} \frac{\mathrm{d} a(x)}{\mathrm{d} a^{\varphi,+}\left(x^{\varphi,+}\right)}=\lambda^{\varphi,+}\left(x^{\varphi,+}\right)\left(-n^{\varphi,+}\left(x^{\varphi,+}\right)\right) .
\end{aligned}
$$


Herein, the real number $\lambda^{\varphi,+}\left(x^{\varphi,+}\right)$ corresponds to:

(i) $x^{\varphi,+} \in$ int Box and $\varphi^{-1}\left(\left\{x^{\varphi,+}\right\}\right)=\{x\}$, then $\lambda^{\varphi,+}\left(x^{\varphi,+}\right)=0$,

(ii) $x^{\varphi,+} \in \partial$ Box, then $\varphi^{-1}\left(\left\{x^{\varphi,+}\right\}\right)=\{x\}$ and $\lambda^{\varphi,+}\left(x^{\varphi,+}\right) \leq 0$,

(iii) $x^{\varphi,+} \in \operatorname{int}$ Box and $\varphi^{-1}\left(\left\{x^{\varphi,+}\right\}\right)=\{x, y\}$ for some $y \in \partial \Omega_{\varepsilon}^{\gamma}$, then $\lambda^{\varphi,+}\left(x^{\varphi,+}\right) \leq 0$.

Similarly, in $x^{\varphi,-}$ we have

$$
\begin{aligned}
T^{\varphi,-}\left(x^{\varphi,-}\right) & \left(+n^{\varphi,-}\left(x^{\varphi,-}\right)\right) \\
& +f_{\text {back }}\left(\left|x^{\varphi,+}-x^{\varphi,-}\right|\right) \frac{x^{\varphi,-}-x^{\varphi,+}}{\left|x^{\varphi,+}-x^{\varphi,-}\right|} \frac{\mathrm{d} a(x)}{\mathrm{d} a^{\varphi,-}\left(x^{\varphi,-}\right)}=\lambda^{\varphi,-}\left(x^{\varphi,-}\right)\left(+n^{\varphi,-}\left(x^{\varphi,-}\right)\right),
\end{aligned}
$$

where $\lambda^{\varphi,-}\left(x^{\varphi,-}\right)$ is characterized analogously to $\lambda^{\varphi,+}\left(x^{\varphi,+}\right)$.

For the derivation of this boundary value problem we refer to [24], Section 2.2.5; the main inspiration behind are calculations performed by Ciarlet and Nečas in [8], Theorem 4.

Thanks to the above equilibrium equations we can interpret the mechanical reality that our model actually describes. From case 2 and the first instance of case 4 we infer static equilibrium in the subbodies of the deformed MBS $\varphi\left(\Omega_{\varepsilon}^{\gamma}\right)$ and on those parts of the deformed inner contact boundary where the lamination is not broken. Case 3 states that any mechanical contact on the deformed outer boundary $\varphi\left(\partial \Omega \cap \partial \Omega_{\varepsilon}^{\gamma}\right)$ is frictionless (selfcontact, contact with the rigid environment, contact with another deformed subbody). Similarly, in the case of a broken lamination in $x \in \Gamma_{C, \varepsilon}^{\gamma}$, i.e. $x^{\varphi,+} \neq x^{\varphi,-}$, the second instance of case 4 implies that the superposition of traction and backdriving force on the deformed inner contact boundaries $\varphi^{ \pm}\left(\Gamma_{C, \varepsilon}^{\gamma}\right)$ is a pressure force - zero in case of no mechanical contact and in case of mechanical contact directed inwards the deformed subbody along the normal. Thus again frictionless mechanical contact on the deformed inner contact boundaries. Note that the backdriving force acting on the deformed surface element around $x^{\varphi,+}$ is directed towards the deformed surface element around $x^{\varphi,-}$. That is, towards the original counterpart involved in the bond. Moreover, the backdriving force is weighted with $\frac{\mathrm{d} a(x)}{\mathrm{d} a^{\varphi},+\left(x^{\varphi},+\right)}$ : to put it simply, if a number of $K$ atomic bonds have been broken on $\mathrm{d} a(x)$ on $\Gamma_{C, \varepsilon}^{\gamma}$, then the backdriving force acting on $\mathrm{d} a^{\varphi,+}\left(x^{\varphi,+}\right)$ is generated by these $K$ broken bonds.

\section{Methodology and mathematical concepts}

Here we outline the mathematical concepts needed for the asymptotic analysis of the total energies $\mathcal{E}_{\varepsilon}^{\gamma}$ associated with MBSs $\Omega_{\varepsilon}^{\gamma}$. Of general interest for the reader might be our novel approximation technique predeformations: a tool to provide approximations for deformations in $\mathrm{SBV}^{p}$ that preserves the kinematic constraints of geometrically nonlinear solid mechanics like $\operatorname{Kin}(\Omega ; \mathbf{B o x})$.

\section{Asymptotics of minimum problems: $\Gamma$-convergence}

Our objective is to analyze the asymptotic behavior of the total energies $\mathcal{E}_{\varepsilon}^{\gamma}$ associated with MBSs $\Omega_{\varepsilon}^{\gamma}$ as $\varepsilon$, i.e. the beam diameter in $\Omega_{\varepsilon}^{\gamma}$, vanishes. Herein, $\Gamma$-convergence (see $[4,10]$ ) appears to be the natural convergence notion. We briefly recall the definition of $\Gamma$-convergence over metric spaces as well as one important property.

Let $(X, d)$ be a metric space, $\mathcal{F}_{k}: X \rightarrow[-\infty, \infty], k \in \mathbb{N}$, be functions, $x \in X$ and $f_{\infty} \in[-\infty, \infty]$.

The value $f_{\infty}$ is said to satisfy the $\Gamma$-liminf-inequality w.r.t. the sequence $\left(\mathcal{F}_{k}\right)_{k}$ in $x$, if for all sequences $\left(x_{k}\right)_{k}$ in $X$ with $x_{k} \rightarrow x$ there holds

$$
f_{\infty} \leq \liminf _{k \rightarrow \infty} \mathcal{F}_{k}\left(x_{k}\right) .
$$

The value $f_{\infty}$ is said to satisfy the $\Gamma$-lim sup-inequality w.r.t. the sequence $\left(\mathcal{F}_{k}\right)_{k}$ in $x$, if there exists a sequence $\left(x_{k}\right)_{k}$ - called recovery sequence - such that $x_{k} \rightarrow x$ and

$$
f_{\infty} \geq \limsup _{k \rightarrow \infty} \mathcal{F}_{k}\left(x_{k}\right)
$$


The sequence $\left(\mathcal{F}_{k}\right)_{k} \Gamma$-converges in $x$ to $f_{\infty}$, in symbols $\left(\Gamma-\lim _{k} \mathcal{F}_{k}\right)(x)=f_{\infty}$, if $f_{\infty}$ satisfies both the $\Gamma$-lim infand the $\Gamma$-lim sup-inequality w.r.t. the sequence $\left(\mathcal{F}_{k}\right)_{k}$ in $x$.

A function $\mathcal{F}_{\infty}: X \rightarrow[-\infty, \infty]$ is said to satisfy the $\Gamma$-lim inf-inequality (respectively the $\Gamma$-lim sup-inequality) w.r.t. the sequence $\left(\mathcal{F}_{k}\right)_{k}$, if in every $x \in X$ the value $\mathcal{F}_{\infty}(x)$ satisfies the $\Gamma$-lim inf-inequality (respectively the $\Gamma$-limsup-inequality) w.r.t. the sequence $\left(\mathcal{F}_{k}\right)_{k}$. The sequence $\left(\mathcal{F}_{k}\right)_{k}$ is said to $\Gamma$-converge to $\mathcal{F}_{\infty}$, in symbols $\Gamma-\lim _{k} \mathcal{F}_{k}=\mathcal{F}_{\infty}$, if there hold both the $\Gamma$-liminf- and the $\Gamma$-limsup-inequality for $\mathcal{F}_{\infty}$ w.r.t. $\left(\mathcal{F}_{k}\right)_{k}$.

In order to prove the $\Gamma$-lim sup-inequality for a sequence of functions, it often suffices to verify it on a dense subset of the underlying metric space (see [4], Rem. 1.29).

Proposition 4.1. Let $\mathcal{F}_{k}, \mathcal{F}_{\infty}: X \rightarrow[-\infty, \infty], k \in \mathbb{N}$, be functions, $x \in X$ and suppose there exists a sequence $\left(z_{j}\right)_{j}$ in $X$ such that:

(i) $z_{j} \rightarrow x$,

(ii) $\mathcal{F}_{\infty}(x) \geq \liminf _{j \rightarrow \infty} \mathcal{F}_{\infty}\left(z_{j}\right)$,

and for every $j \in \mathbb{N}$ :

(iii) the value $\mathcal{F}_{\infty}\left(z_{j}\right)$ satisfies the $\Gamma$-limsup-inequality w.r.t. the sequence $\left(\mathcal{F}_{k}\right)_{k}$ in $z_{j}$.

Then also $\mathcal{F}_{\infty}(x)$ satisfies the $\Gamma$-limsup-inequality w.r.t. the sequence $\left(\mathcal{F}_{k}\right)_{k}$ in $x$.

\section{Special functions of bounded variation}

Until the end of the present section let $\Omega$ be an open and bounded subset of $\mathbb{R}^{N}$ and Box a compact subset of $\mathbb{R}^{N}$ with nonempty interior. For a (Borel-) measurable subset $U$ of $\Omega$ and a finite $\mathbb{M}^{N}$-valued Radon-measure $\mu$ we write $\mu\llcorner U$ for the restriction of $\mu$ to $U$, i.e. $(\mu\llcorner U)(A):=\mu(U \cap A)$ for every measurable subset $A$ of $\Omega$.

The space $\operatorname{BV}\left(\Omega ; \mathbb{R}^{N}\right)$ of functions of bounded variation in $\Omega$ is the set of all $\varphi \in \mathrm{L}^{1}\left(\Omega ; \mathbb{R}^{N}\right)$ whose distributional derivative can be represented by a finite $\mathrm{M}^{N}$-valued Radon-measure $\operatorname{D} \varphi$. For $\operatorname{BV}\left(\Omega ; \mathbb{R}^{N}\right)$-functions $\varphi$, the approximate discontinuity set $S_{\varphi}$ is countably $\mathcal{H}^{N-1}$-rectifiable, i.e. is up to a set of $\mathcal{H}^{N-1}$-measure 0 contained in the union of countably many Lipschitz-hypersurfaces. Furthermore, the derivative $\mathrm{D} \varphi$ can be decomposed into

$$
\mathrm{D} \varphi=\mathrm{D}^{a} \varphi+\mathrm{D}^{j} \varphi+\mathrm{D}^{c} \varphi
$$

Herein, $\mathrm{D}^{a} \varphi=\nabla \varphi$ vol $\left\llcorner\Omega\right.$ is the absolutely continuous part of $\mathrm{D} \varphi$ w.r.t. vol $L \Omega$ and $\nabla \varphi \in \mathrm{L}^{1}\left(\Omega ; \mathbb{M}^{N}\right)$ the corresponding density function. Every $\varphi \in \operatorname{BV}\left(\Omega ; \mathbb{R}^{N}\right)$ is a.e. approximately differentiable and the approximate differential ap $\mathrm{D} \varphi$ equals the density $\nabla \varphi$. The quantity $\mathrm{D}^{j} \varphi=\left(\varphi^{+}-\varphi^{-}\right) \otimes \nu_{\varphi} \mathcal{H}^{N-1}\left\llcorner S_{\varphi}\right.$ is the jump part of $\mathrm{D} \varphi$ : the (unique) triplet of Borel-functions $\left(\varphi^{+}, \varphi^{-}, \nu_{\varphi}\right): S_{\varphi} \rightarrow \mathbb{R}^{N} \times \mathbb{R}^{N} \times S^{N-1}$ satisfies in $\mathcal{H}^{N-1}$-a.e. point $x_{0} \in S_{\varphi}$

$$
\lim _{r \rightarrow 0} \frac{1}{\operatorname{vol} B_{r}^{ \pm}\left(x_{0}, \nu_{\varphi}\left(x_{0}\right)\right)} \int_{B_{r}^{ \pm}\left(x_{0}, \nu_{\varphi}\left(x_{0}\right)\right)}\left|\varphi(x)-\varphi^{ \pm}\left(x_{0}\right)\right| \mathrm{d} x=0,
$$

where $B_{r}^{ \pm}\left(x_{0}, \nu_{\varphi}\left(x_{0}\right)\right):=\left\{x: x \in B_{r}\left(x_{0}\right), \pm \nu_{\varphi}\left(x_{0}\right) \cdot\left(x-x_{0}\right)>0\right\}$. The quantity $\mathrm{D}^{c} \varphi$ is called the Cantor part of $\mathrm{D} \varphi$.

The $\mathrm{BV}\left(\Omega ; \mathbb{R}^{N}\right)$-functions $\varphi$ whose derivatives have zero Cantor part $\mathrm{D}^{c} \varphi=0$ are called the special functions of bounded variation in $\Omega$ and form the $\operatorname{space} \operatorname{SBV}\left(\Omega ; \mathbb{R}^{N}\right)$. For results on $\operatorname{SBV}$ we refer to the standard reference [2]. Of particular importance to us are the following two propositions on SBV.

Proposition 4.2. Let $K$ be a compact subset of $\mathbb{R}^{N}$ such that $\mathcal{H}^{N-1}(K)<\infty$ and $\varphi \in \operatorname{SBV}\left(\Omega \backslash K ; \mathbb{R}^{N}\right) \cap$ $\mathrm{L}^{\infty}\left(\Omega \backslash K ; \mathbb{R}^{N}\right)$. Write $\bar{\varphi} \in \mathrm{L}^{1}\left(\Omega ; \mathbb{R}^{N}\right)$ for the identification of $\varphi$ in $\mathrm{L}^{1}\left(\Omega ; \mathbb{R}^{N}\right)$. Then

$$
\bar{\varphi} \in \operatorname{SBV}\left(\Omega ; \mathbb{R}^{N}\right), \quad \nabla \bar{\varphi}=\nabla \varphi \text { a.e. in } \Omega \quad \text { and } \quad S_{\bar{\varphi}} \subseteq S_{\varphi} \cup K \text {. }
$$

Proposition 4.2 is only a minor generalization of [2], Proposition 4.4, and can be proved by following the very lines of the proof in the reference given. 
Proposition 4.3. Let $G$ be an open and bounded subset of $\mathbb{R}^{N}, \Phi: G \rightarrow \Omega$ be invertible and such that:

(i) $\Phi$ is continuous and of class $\mathrm{W}^{1, \infty}\left(G ; \mathbb{R}^{N}\right)$,

(ii) $\Phi^{-1}$ is Lipschitz,

(iii) $\operatorname{det} \mathrm{D} \Phi>0$ a.e. in $G$.

Then for all $\varphi \in \operatorname{SBV}\left(\Omega ; \mathbb{R}^{N}\right)$ the function $\psi:=\varphi \circ \Phi$ is in $\operatorname{SBV}\left(G ; \mathbb{R}^{N}\right)$ and there holds

$$
\nabla \psi=(\nabla \varphi \circ \Phi) \cdot \mathrm{D} \Phi \text { a.e. in } G \text { and } S_{\psi}=\Phi^{-1}\left(S_{\varphi}\right) \text {. }
$$

This result was indicated in [2] as Exercise 4.5 with more restrictive regularity assumptions on the coordinate transformation. A complete proof is given in [24], Proposition 3.23.

For $1 \leq p<\infty$ we define the space

$$
\operatorname{SBV}^{p}\left(\Omega ; \mathbb{R}^{N}\right):=\left\{\varphi: \varphi \in \operatorname{SBV}\left(\Omega ; \mathbb{R}^{N}\right), \nabla \varphi \in \mathrm{L}^{p}\left(\Omega ; \mathbb{M}^{N}\right), \mathcal{H}^{N-1}\left(S_{\varphi}\right)<\infty\right\}
$$

Let $\varphi_{k}, \varphi$ be elements of $\operatorname{SBV}^{p}\left(\Omega ; \mathbb{R}^{N}\right)$. We say that the sequence $\left(\varphi_{k}\right)_{k}$ weakly converges to $\varphi$ in $\operatorname{SBV}^{p}\left(\Omega ; \mathbb{R}^{N}\right)$, in symbols $\varphi_{k} \rightarrow \varphi$ in $\operatorname{SBV}^{p}\left(\Omega ; \mathbb{R}^{N}\right)$, if

$$
\varphi_{k} \rightarrow \varphi \quad \text { in } \mathrm{L}^{1}\left(\Omega ; \mathbb{R}^{N}\right), \quad \nabla \varphi_{k} \rightarrow \nabla \varphi \quad \text { in } \mathrm{L}^{p}\left(\Omega ; \mathbb{M}^{N}\right) \quad \text { and } \quad \sup _{k \in \mathbb{N}} \mathcal{H}^{N-1}\left(S_{\varphi_{k}}\right)<\infty
$$

\section{Compactness and lower semicontinuity in $\mathrm{SBV}^{p}$}

In [8] Ciarlet and Nečas proved stability of the Ciarlet-Nečas condition under weak convergence in the Sobolev-space $\mathrm{W}^{1, p}\left(\Omega ; \mathbb{R}^{N}\right)$ for $p>N$. Recently, Giacomini and Ponsiglione showed in [15] that the CiarletNečas condition is also stable under weak convergence in $\operatorname{SBV}^{p}\left(\Omega ; \mathbb{R}^{N}\right)$ (see [15], Thm. 4.4, and [2], Cor. 5.31).

Theorem 4.4. Let $\left(\varphi_{k}\right)_{k}$ and $\varphi$ be in $\operatorname{SBV}^{p}\left(\Omega ; \mathbb{R}^{N}\right), p>N$, such that $\varphi_{k}$ satisfies the Ciarlet-Nečas condition for every $k \in \mathbb{N}$ and $\varphi_{k} \rightarrow \varphi$ in $\operatorname{SBV}^{p}\left(\Omega ; \mathbb{R}^{N}\right)$. Then $\varphi$ satisfies the Ciarlet-Nečas condition.

Proposition 4.5. Let $M \subseteq \operatorname{SBV}^{p}\left(\Omega ; \mathbb{R}^{N}\right), p>N$, be closed w.r.t. weak convergence in $\operatorname{SBV}^{p}\left(\Omega ; \mathbb{R}^{N}\right)$ and such that $\operatorname{Kin}(\Omega ; \mathbf{B o x}) \cap M$ is nonempty. Let $W$ satisfy $(W 1), \ldots,(W 3), \theta$ be in accordance with $(\theta 1), \ldots,(\theta 3)$ and $F$ with $(F 1),(F 2)$. Moreover, let $\phi: \mathbb{R}^{N} \rightarrow[0, \infty)$ be even, positively 1-homogeneous, convex and such that it permits a positive uniform bound from below on $S^{N-1}$. Set

$$
\begin{aligned}
& \mathcal{F}: \operatorname{SBV}^{p}\left(\Omega ; \mathbb{R}^{N}\right) \cap \operatorname{Kin}(\Omega ; \operatorname{Box}) \rightarrow(-\infty, \infty] \\
& \mathcal{F}(\varphi):= \begin{cases}\int_{\Omega} W(\nabla \varphi) \mathrm{d} x+\int_{S_{\varphi}} \phi\left(\nu_{\varphi}\right) \theta\left(\left|\varphi^{+}-\varphi^{-}\right|\right) \mathrm{d} \mathcal{H}^{N-1}-\int_{\Omega} F(x, \varphi(x)) \mathrm{d} x & \text { if } \varphi \in M, \\
\infty, & \text { otherwise. }\end{cases}
\end{aligned}
$$

Then

(i) $\mathcal{F}$ is sequentially lower semicontinuous w.r.t. strong $\mathrm{L}^{1}\left(\Omega ; \mathbb{R}^{N}\right)$-convergence,

(ii) there is a minimizer of $\mathcal{F}$ in $M \cap \operatorname{Kin}(\Omega ; \mathbf{B o x})$.

Proof. As concerns the first assertion, let there be given a sequence $\left(\varphi_{k}\right)_{k}$ and $\varphi$ in $\operatorname{SBV}^{p}\left(\Omega ; \mathbb{R}^{N}\right) \cap \operatorname{Kin}(\Omega ; \operatorname{Box})$ such that $\varphi_{k} \rightarrow \varphi$ in $\mathrm{L}^{1}\left(\Omega ; \mathbb{R}^{N}\right)$. Without loss of generality we may assume $\infty>\lim \inf _{k} \mathcal{F}\left(\varphi_{k}\right)=\lim _{m} \mathcal{F}\left(\varphi_{k}(m)\right)$ for an appropriate subsequence. Then the assumptions on $W, \theta, \phi$ and $F$ together with the Box-constraint imply

$$
\infty>\sup _{m \in \mathbb{N}}\left\{\left\|\varphi_{k(m)}\right\|_{L^{\infty}\left(\Omega ; \mathbb{R}^{N}\right)}+\left\|\nabla \varphi_{k(m)}\right\|_{L^{p}\left(\Omega ; \mathbb{M}^{N}\right)}+\mathcal{H}^{N-1}\left(S_{\varphi_{k(m)}}\right)\right\} .
$$

From Ambrosio's compactness theorem [2], Theorems 4.7 and 4.8, we infer the existence of a subsequence of $\left(\varphi_{k(m)}\right)_{m}$ (not relabeled) that weakly converges to $\varphi$ in $\operatorname{SBV}^{p}\left(\Omega ; \mathbb{R}^{N}\right)$. Moreover, the Jacobian determinant 
is weakly $\mathrm{L}^{r}(\Omega)$-continuous w.r.t. weak convergence in $\operatorname{SBV}^{p}\left(\Omega ; \mathbb{R}^{N}\right)$ for $r \in\left[1, \frac{p}{N}\right)$ (see [2], Cor. 5.31). The assertion then follows from standard lower semicontinuity results on polyconvex integral functionals, from lower semicontinuity properties of surface integral functionals (see [2], Thm. 5.22, Ex. 5.23) and the $\mathrm{L}^{1}$-continuity of $\psi \mapsto \int_{\Omega} F(x, \psi(x))$ by $(F 1)$.

The second assertion is proved similarly upon taking into account the stability of the Ciarlet-Nečas condition w.r.t. weak convergence in $\operatorname{SBV}^{p}\left(\Omega ; \mathbb{R}^{N}\right)$ by Theorem 4.4.

Remark 4.1. In order to obtain sequential compactness in $\mathrm{SBV}^{p}$ w.r.t. weak convergence in $\mathrm{SBV}^{p}$, it is crucial to have a uniform $\mathrm{L}^{\infty}$-bound, see the discussion in [2], Remark 4.9. The sole purpose of our confinement condition Box is to provide such a uniform $\mathrm{L}^{\infty}$-bound. From the mathematical point of view this is a strong restriction, while not unnatural in terms of mechanics, $c f$. Remark 3.4. Similar compactness without a previously imposed $\mathrm{L}^{\infty}$-bound can only be expected in GSBV, the generalized special functions of bounded variation. However, for this first contribution to the homogenization of largely deformed MBSs we chose to confine ourselves to this simpler case and to avoid the more difficult GSBV-context.

Proposition 4.6. Let $\left(\varphi_{k}\right)_{k}$ be a sequence in $\operatorname{SBV}^{p}\left(\Omega ; \mathbb{R}^{N}\right), 1 \leq p<\infty$, satisfying $\sup _{k}\left\|\varphi_{k}\right\|_{L^{\infty}\left(\Omega ; \mathbb{R}^{N}\right)}<\infty$ and for an $i \in\{1, \ldots, N\}$

$$
\nu_{\varphi_{k}, i}=0 \quad \mathcal{H}^{N-1} \text {-a.e. on } S_{\varphi_{k}} \quad \text { for all } k \in \mathbb{N} .
$$

In case there is $\varphi \in \operatorname{SBV}^{p}\left(\Omega ; \mathbb{R}^{N}\right)$ such that $S_{\varphi} \neq \emptyset$ and $\varphi_{k} \rightarrow \varphi$ in $\operatorname{SBV}^{p}\left(\Omega ; \mathbb{R}^{N}\right)$ one has

$$
\nu_{\varphi, i}=0 \quad \mathcal{H}^{N-1} \text {-a.e. on } S_{\varphi} .
$$

Proof. Weak convergence in $\operatorname{SBV}^{p}\left(\Omega ; \mathbb{R}^{N}\right)$ together with $\sup _{k}\left\|\varphi_{k}\right\|_{\mathrm{L}^{\infty}\left(\Omega ; \mathbb{R}^{N}\right)}<\infty$ implies weak*-convergence of the derivatives $\left(\mathrm{D} \varphi_{k}\right)_{k}$ to $\mathrm{D} \varphi$ in the sense of Radon measures, furthermore by definition it is $\nabla \varphi_{k} \rightarrow \nabla \varphi$ in $\mathrm{L}^{p}\left(\Omega ; \mathrm{M}^{N}\right)$. We infer $\mathrm{D}^{j} \varphi_{k} \stackrel{*}{\rightarrow} \mathrm{D}^{j} \varphi$ in the sense of Radon-measures, thus in particular

$$
\nu_{\varphi_{k}, i}\left(\varphi_{k}^{+}-\varphi_{k}^{-}\right) \mathcal{H}^{N-1}\left\llcornerS _ { \varphi _ { k } } \stackrel { * } { \rightarrow } \nu _ { \varphi , i } ( \varphi ^ { + } - \varphi ^ { - } ) \mathcal { H } ^ { N - 1 } \left\llcorner S_{\varphi} \quad\right.\right. \text { in the sense of Radon-measures. }
$$

The left hand side is the zero measure for every $k \in \mathbb{N}$ due to $\nu_{\varphi_{k}, i}$ being zero. Therefore, $\nu_{\varphi, i}\left(\varphi^{+}-\right.$ $\left.\varphi^{-}\right) \mathcal{H}^{N-1}\left\llcorner S_{\varphi}\right.$ is the zero measure and we finish the proof by noticing that $\varphi^{+}-\varphi^{-} \neq 0 \mathcal{H}^{N-1}$-a.e. on $S_{\varphi}$.

\section{Predeformations}

The natural kinematic constraints of all problems in geometrically nonlinear solid mechanics are preservation of local orientation, noninterpenetration of matter, i.e. injectivity, and even a confinement condition. In other words, deformations of the $N$-dimensional open and bounded solid body $\Omega$ - be it elastic, plastic or brittle must always be elements of the set $\operatorname{Kin}(\Omega ; \mathbf{B o x})$. Now many mechanical problems that allow for jumps in the deformations across $(N-1)$-dimensional crack surfaces are formulated over the set $\operatorname{SBV}^{p}\left(\Omega ; \mathbb{R}^{N}\right)$ - including ours and several of the references given. When imposing the natural kinematic constraints $\operatorname{Kin}(\Omega ; \mathbf{B o x})$ on such a model, analysis often requires approximations inside the set of attainable deformations $\operatorname{SBV}^{p}\left(\Omega ; \mathbb{R}^{N}\right) \cap$ $\operatorname{Kin}(\Omega ;$ Box $)$. More precisely, given a $\varphi \in \operatorname{SBV}^{p}\left(\Omega ; \mathbb{R}^{N}\right) \cap \operatorname{Kin}(\Omega ;$ Box $)$, how to find a $\psi$ in the same set that is "close" to $\varphi$ (e.g. in the $\mathrm{L}^{1}$-distance) while its discontinuity set $S_{\psi}$ has a "certain" geometry (e.g. contained in a given set)? The approximation techniques used so far in the mathematical literature (see e.g. $[9,14])$ are based on reflection arguments, or simply "flatten" the deformation to 0 wherever it appears useful. But such techniques are incompatible with the kinematic constraints $\operatorname{Kin}(\Omega ;$ Box $)$ and therefore unsuitable here. A way to overcome this dilemma is the use of what we call predeformations.

Definition 4.7. Let $U$ and $G$ be open and bounded subsets of $\mathbb{R}^{N}$. A bijective mapping $\Phi: G \rightarrow U$ is called a predeformation, if:

(i) $\Phi$ is continuous and of class $\mathrm{W}^{1, \infty}\left(G ; \mathbb{R}^{N}\right)$,

(ii) $\Phi^{-1}$ is Lipschitz,

(iii) $\operatorname{det} \mathrm{D} \Phi>0$ a.e. in $G$. 

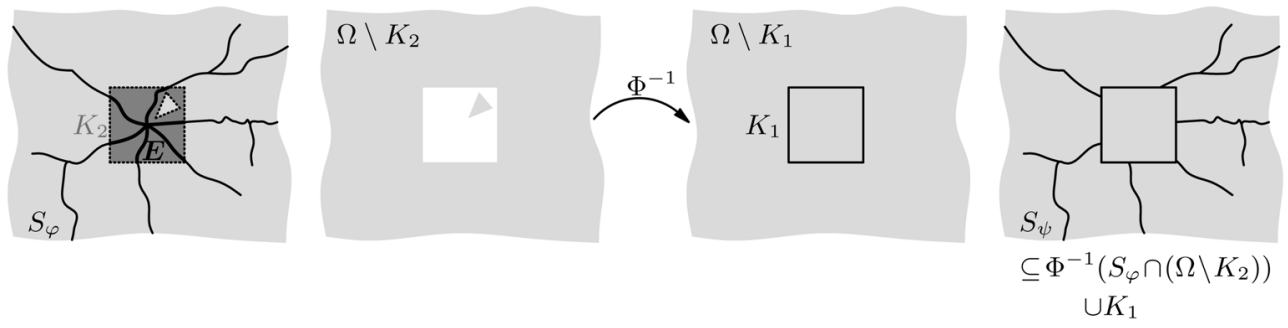

Figure 3. Approximation by predeformations: Cutting out what doesn't fit.

The idea behind predeformations is as simple as intuitive. Instead of approximating the deformation $\varphi$ by manipulating its values we "predeform" the underlying domain instead. In particular we predeform the discontinuity set $S_{\varphi}$ until it shows the desired geometry. A major strength of predeformations is that they can be used to "cut out" undesirable properties of $\varphi$. Before illustrating this by means of a short example, we state how predeformations act on $\operatorname{SBV}^{p}\left(\Omega ; \mathbb{R}^{N}\right) \cap \operatorname{Kin}(\Omega ;$ Box $)$-deformations.

Proposition 4.8. Let $G$ be an open and bounded subset of $\mathbb{R}^{N}$ and $\Omega^{\prime}$ be an open subset of $\Omega$. Suppose $K$ is a compact set in $\mathbb{R}^{N}$ with $\mathcal{H}^{N-1}(K)<\infty$ and let $\Phi: G \backslash K \rightarrow \Omega^{\prime}$ be a predeformation. For $\varphi \in$ $\operatorname{SBV}^{p}\left(\Omega ; \mathbb{R}^{N}\right) \cap \operatorname{Kin}(\Omega ; \operatorname{Box})$, where $1 \leq p<\infty$, set $\psi:=\varphi \circ \Phi \in \mathrm{L}^{1}\left(G \backslash K ; \mathbb{R}^{N}\right)$ and write $\bar{\psi}$ for the identification of $\psi$ in $\mathrm{L}^{1}\left(G ; \mathbb{R}^{N}\right)$. Then

$$
\bar{\psi} \in \mathrm{SBV}^{p}\left(G ; \mathbb{R}^{N}\right) \cap \operatorname{Kin}(G ; \mathbf{B o x}), \nabla \bar{\psi}=(\nabla \varphi \circ \Phi) \cdot \mathrm{D} \Phi \quad \text { and } \quad S_{\bar{\psi}} \subseteq \Phi^{-1}\left(S_{\varphi} \cap \Omega^{\prime}\right) \cup K
$$

If $K=\emptyset$, then $S_{\bar{\psi}}=\Phi^{-1}\left(S_{\varphi} \cap \Omega^{\prime}\right)$.

Proof. Obviously $\left.\varphi\right|_{\Omega^{\prime}} \in \operatorname{SBV}^{p}\left(\Omega^{\prime} ; \mathbb{R}^{N}\right) \cap \operatorname{Kin}\left(\Omega^{\prime} ;\right.$ Box $), \quad c f$. Remark 3.2. Proposition 4.3 implies $\psi \in$ $\operatorname{SBV}\left(G \backslash K ; \mathbb{R}^{N}\right)$ and

$$
\nabla \psi=(\nabla \varphi \circ \Phi) \cdot \mathrm{D} \Phi \quad \text { as well as } \quad S_{\psi}=\Phi^{-1}\left(S_{\varphi} \cap \Omega^{\prime}\right) .
$$

It is easily seen that $\nabla \psi \in \mathrm{L}^{p}\left(G \backslash K ; \mathbb{M}^{N}\right)$ and $\mathcal{H}^{N-1}\left(S_{\psi}\right)<\infty$, hence $\psi \in \operatorname{SBV}^{p}\left(G \backslash K\right.$; $\left.\mathbb{R}^{N}\right)$. Since $\Phi$ is a predeformation, by (4.3) we infer $\operatorname{det} \nabla \psi>0$ a.e., and from a.e.-injectivity of $\varphi$ and bijectivity of $\Phi$ moreover the a.e.-injectivity of $\psi$. Trivially $\psi(x) \in \operatorname{Box}$ a.e. and we conclude $\psi \in \operatorname{SBV}^{p}\left(G \backslash K ; \mathbb{R}^{N}\right) \cap \operatorname{Kin}(G \backslash K ; \operatorname{Box})$.

Proposition 4.2 (note that the Box-constraint provides an $\mathrm{L}^{\infty}$-bound on $\psi$ ) gives $\bar{\psi} \in \mathrm{SBV}^{p}\left(G ; \mathbb{R}^{N}\right)$ and in view of (4.3) Proposition 4.2 yields furthermore $\nabla \bar{\psi}=(\nabla \varphi \circ \Phi) \cdot \mathrm{D} \Phi$ and $S_{\bar{\psi}} \subseteq \Phi^{-1}\left(S_{\varphi} \cap \Omega^{\prime}\right) \cup K$. The proof is finished upon realizing that $\operatorname{vol} K=0$ allows to identify $\operatorname{Kin}(G \backslash K$; Box $)$ and $\operatorname{Kin}(G ; \operatorname{Box})$.

Example 4.1. Consider a deformation $\varphi \in \operatorname{SBV}^{p}\left(\Omega ; \mathbb{R}^{N}\right) \cap \operatorname{Kin}(\Omega ;$ Box $)$ showing certain "undesirable" properties concentrated on a subset $E$ of $\Omega$ of Hausdorff-dimension $N-1$. We want to approximate $\varphi$ with a deformation $\psi \in \operatorname{SBV}^{p}\left(\Omega ; \mathbb{R}^{N}\right) \cap \operatorname{Kin}(\Omega ;$ Box $)$ that does not possess these undesirable properties. Assume that $E$ can be covered with a compact subset $K_{2}$ of $\Omega$ such that such that $E \subseteq \operatorname{int} K_{2}$. By "cutting out" $K_{2}$ of $\Omega$ we could get rid of $E$ and its undesirable properties, but this would result in a "hole" in $\Omega$. However, suppose we can construct a predeformation $\Phi$ such that its inverse "closes" the hole in $\Omega$ and leaves just a compact "seam" $K_{1}$ of Hausdorff-dimension $(N-1)$, i.e. $\Phi: \Omega \backslash K_{1} \rightarrow \Omega \backslash K_{2}$ (e.g. in Fig. $3 \Phi^{-1}$ blows up the little triangle we took out of $K_{2}$ ). Then the deformation $\psi:=\varphi \circ \Phi$ provides the approximation we want: by Proposition 4.8 it is like $\varphi$ element of $\operatorname{SBV}^{p}\left(\Omega ; \mathbb{R}^{N}\right) \cap \operatorname{Kin}(\Omega ; \mathbf{B o x})$ and does not show the undesirable properties of $\varphi$ since $E \cap \Phi\left(\Omega \backslash K_{1}\right)=\emptyset$. If we can make the volume of the set on which $\Phi$ differs from the identity arbitrarily small, then also the $\mathrm{L}^{1}$-distance between $\psi$ and $\varphi$ vanishes. Thus $\psi$ approximates $\varphi$ in the norm-topology of $\mathrm{L}^{1}\left(\Omega ; \mathbb{R}^{N}\right)$.

Remark 4.2. In [11], Lemma 4.1, Dal Maso and Lazzaroni - studying quasistatic crack growth in finite elasticity under kinematic constraints like ours - independently and contemporaneously employed piecewise 
linear predeformations ("stretching" in their nomenclature) to avoid reflection arguments when manipulating the discontinuity sets of kinematically admissible $\operatorname{SBV}^{p}\left(\Omega ; \mathbb{R}^{N}\right)$-deformations.

Remark 4.3. In general, the discontinuity sets of $\operatorname{SBV}^{p}\left(\Omega ; \mathbb{R}^{N}\right)$-deformations are merely countably $\mathcal{H}^{N-1}$ rectifiable, what makes them quite difficult to deal with. In some situations one has to restrict the analysis to elements of $\operatorname{SBV}^{p}\left(\Omega ; \mathbb{R}^{N}\right)$ with more regular discontinuity sets. Then, many of the previously presented concepts in $\operatorname{SBV}^{p}\left(\Omega ; \mathbb{R}^{N}\right)$ become remarkably simple (see [24], Sect. 3.3.3): let $\varphi \in \operatorname{SBV}^{p}\left(\Omega ; \mathbb{R}^{N}\right), p>N$, be such that $S_{\varphi}$ is contained in a piecewise $\mathrm{C}^{1}$-hypersurface $S$ with normal field $\nu_{S}$ :

(i) For $\mathcal{H}^{N-1}$-a.e. $x_{0} \in S_{\varphi}$ it is $\left(\varphi^{+}\left(x_{0}\right), \varphi^{-}\left(x_{0}\right), \nu_{\varphi}\left(x_{0}\right)\right)=\left(\mathrm{T}^{+} \varphi\left(x_{0}\right), \mathrm{T}^{-} \varphi\left(x_{0}\right), \nu_{S}\left(x_{0}\right)\right)$, where $\mathrm{T}^{ \pm} \varphi\left(x_{0}\right)=$ $\lim _{n} \varphi\left(x_{n}\right), x_{n} \in \Omega$ on the side of $S$ which $\pm \nu_{S}\left(x_{0}\right)$ points to and $x_{n} \rightarrow x_{0}$. This allows us to define $\left(\varphi^{+}, \varphi^{-}, \nu_{\varphi}\right)$ on the whole of $S$ by identification with $\left(\mathrm{T}^{+} \varphi, \mathrm{T}^{-} \varphi, \nu_{S}\right)$ and the jump part of $\mathrm{D} \varphi$ becomes

$$
\mathrm{D}^{j} \varphi=\left(\varphi^{+}-\varphi^{-}\right) \otimes \nu_{S} \mathcal{H}^{N-1}\llcorner(S \cap \Omega) .
$$

(ii) Let $G$ be an open and bounded set in $\mathbb{R}^{N}, H$ a piecewise $\mathrm{C}^{1}$-hypersurface with normal field $\nu_{H}$ and $\Phi: G \rightarrow \Omega$ be a coordinate transformation in the sense of Proposition 4.3. Assume that $\Phi^{-1}(S \cap \Omega)=$ $H \cap G$ and $\Phi$ maps the side of $H$ which $\pm \nu_{H}$ points to onto the side of $S$ which $\pm \nu_{S}$ points to. Then the deformation $\psi:=\varphi \circ \Phi \in \operatorname{SBV}^{p}\left(G ; \mathbb{R}^{N}\right)$ satisfies $S_{\psi} \subseteq H$ and

$$
\mathrm{D}^{j} \psi=\left(\varphi^{+}-\varphi^{-}\right) \circ \Phi \otimes \nu_{H} \mathcal{H}^{N-1}\llcorner(H \cap G)
$$

To conclude this section, let us define the vector space $\mathcal{V}^{p}\left(\Omega ; \mathbb{R}^{N}\right)$ as the set of deformations $\varphi \in \mathrm{L}^{p}\left(\Omega ; \mathbb{R}^{N}\right)$, for each of which exists a polyhedral set $P$ such that $\varphi \in \mathrm{W}^{1, p}\left(\Omega \backslash P ; \mathbb{R}^{N}\right)$. Clearly $\mathcal{V}^{p}\left(\Omega ; \mathbb{R}^{N}\right) \subseteq \operatorname{SBV}^{p}\left(\Omega ; \mathbb{R}^{N}\right)$.

Remark 4.4. Cortesani and Toader showed in $[9]$ that $\mathcal{V}^{p}\left(\Omega ; \mathbb{R}^{N}\right)$ has excellent density properties in $\operatorname{SBV}^{p}\left(\Omega ; \mathbb{R}^{N}\right)$. But it is not clear and remains an open question whether similar density results can be established for $\mathcal{V}^{p}\left(\Omega ; \mathbb{R}^{N}\right) \cap \operatorname{Kin}(\Omega ; \mathbf{B o x})$ in $\operatorname{SBV}^{p}\left(\Omega ; \mathbb{R}^{N}\right) \cap \operatorname{Kin}(\Omega ; \mathbf{B o x})$.

\section{Analysis of the mathematical model}

The rest of the article is concerned with the asymptotic analysis of the mathematical model for the MBSs $\Omega_{\varepsilon}^{\gamma}$ we introduced in Section 3. We start with a proof of Theorem 3.4.

Proof of Theorem 3.4. The statement follows immediately from Proposition 4.5 (wherein we set $\phi(v):=|v|$, $M=\mathrm{W}^{1, p}\left(\Omega_{\varepsilon}^{\gamma} ; \mathbb{R}^{3}\right)$ and use that $\mathrm{W}^{1, p}\left(\Omega_{\varepsilon}^{\gamma} ; \mathbb{R}^{3}\right)$ as a subset of $\operatorname{SBV}^{p}\left(\Omega ; \mathbb{R}^{3}\right)$ is closed w.r.t. weak convergence in $\left.\operatorname{SBV}^{p}\left(\Omega ; \mathbb{R}^{3}\right)\right)$.

\section{Homogenization by $\Gamma$-convergence}

The $\Gamma$-convergence analysis of the total energies $\mathcal{E}_{\varepsilon}^{\gamma}$ for vanishing $\varepsilon$ requires their extension to one common metric space. A natural candidate is $\operatorname{SBV}^{p}\left(\Omega ; \mathbb{R}^{3}\right) \cap \operatorname{Kin}(\Omega ; \mathbf{B o x})$ equipped with the strong $\mathrm{L}^{1}\left(\Omega ; \mathbb{R}^{3}\right)$-topology. Since a MBS $\Omega_{\varepsilon}^{\gamma}$ can only undergo deformations with jumps across the inner contact boundary $\Gamma_{C, \varepsilon}^{\gamma}$, we must extend $\mathcal{E}_{\varepsilon}^{\gamma}$ to $\operatorname{SBV}^{p}\left(\Omega ; \mathbb{R}^{3}\right) \cap \operatorname{Kin}(\Omega ;$ Box $)$ by $\infty$. Moreover, $\Gamma$-convergence is stable under continuous perturbations [4], Remark 1.7. Since we assumed in $(F 1)$ the $\mathrm{L}^{1}\left(\Omega ; \mathbb{R}^{3}\right)$-continuity of the load term $\psi \mapsto \int_{\Omega} F(x, \psi(x)) \mathrm{d} x$ it can be omitted in a $\Gamma$-convergence study of the extended total energies. Consequently, it suffices to study the extended total energies associated with MBSs $\Omega_{\varepsilon}^{\gamma}$ defined as

$$
\begin{aligned}
\mathcal{F}_{\varepsilon}^{\gamma}: \mathrm{SBV}^{p}\left(\Omega ; \mathbb{R}^{3}\right) \cap \operatorname{Kin}(\Omega ; \mathbf{B o x}) \rightarrow & {[0, \infty], } \\
& \mathcal{F}_{\varepsilon}^{\gamma}(\varphi):= \begin{cases}\int_{\Omega} W(\nabla \varphi) \mathrm{d} x+\int_{S_{\varphi}} \theta\left(\left|\varphi^{+}-\varphi^{-}\right|\right) \mathrm{d} \mathcal{H}^{2} \\
\infty & \text { if } \varphi \in \mathrm{W}^{1, p}\left(\Omega_{\varepsilon}^{\gamma} ; \mathbb{R}^{3}\right), \\
\text { else. }\end{cases}
\end{aligned}
$$


In order to reduce the technical efforts in the $\Gamma$-convergence analysis of $\mathcal{F}_{\varepsilon}^{\gamma}$ for $\varepsilon$ tending to zero, we consider particular vanishing sequences of positive real numbers $\left(\varepsilon_{k}\right)_{k}$. More specifically, we demand $\left(\varepsilon_{k}\right)_{k}$ to be a refining sequence, in the sense that $\frac{\varepsilon_{k}}{\varepsilon_{k+1}} \in \mathbb{N}$ for all $k \in \mathbb{N}$.

\section{Statement of the homogenization results}

We can characterize the asymptotic behavior of the MBSs $\Omega_{\varepsilon}^{\gamma}$ by means of the following homogenization results.

Theorem 5.1 (homogenization of the MBS with zero cord-angle). Let $\Omega, \Omega_{\varepsilon_{k}}^{0}, \Gamma_{C, \varepsilon_{k}}^{0}$ be given like in Definition of Geometry 3.1. Suppose that the elastic energy density $W$ satisfies $(W 1), \ldots,(W 4)$ and that the surface energy density $\theta$ obeys $(\theta 1), \ldots,(\theta 3)$. Define the homogenized total energy

$$
\begin{aligned}
\mathcal{F}_{\mathrm{Hom}}^{0}: \mathrm{SBV}^{p}\left(\Omega ; \mathbb{R}^{3}\right) \cap \operatorname{Kin}(\Omega ; \mathbf{B o x}) & \rightarrow[0, \infty], \\
\mathcal{F}_{\mathrm{Hom}}^{0}(\varphi) & := \begin{cases}\int_{\Omega} W(\nabla \varphi) \mathrm{d} x+\int_{S_{\varphi}} \phi\left(\nu_{\varphi}\right) \theta\left(\left|\varphi^{+}-\varphi^{-}\right|\right) \mathrm{d} \mathcal{H}^{2} \\
\infty & \text { if } \nu_{\varphi, 1}=0 \mathcal{H}^{2} \text {-a.e. on } S_{\varphi},\end{cases}
\end{aligned}
$$

where the anisotropy factor $\phi$ generated by the microstructure $\mathcal{D}_{0}$ is given by

$$
\phi: S^{2} \rightarrow[0, \infty), \quad \phi(v):=\left|v_{2}\right|+\left|v_{3}\right| .
$$

Then for the sequence $\left(\mathcal{F}_{\varepsilon_{k}}^{0}\right)_{k}$ and the homogenized total energy $\mathcal{F}_{\mathrm{Hom}}^{0}$ there holds w.r.t. strong $\mathrm{L}^{1}\left(\Omega ; \mathbb{R}^{3}\right)$ convergence

the $\Gamma$-lim inf -inequality in every element of $\operatorname{SBV}^{p}\left(\Omega ; \mathbb{R}^{3}\right) \cap \operatorname{Kin}(\Omega ; \mathbf{B o x})$, and moreover

the $\Gamma$-lim sup-inequality in at least all $\varphi \in \operatorname{SBV}^{p}\left(\Omega ; \mathbb{R}^{3}\right) \cap \operatorname{Kin}(\Omega ;$ Box $)$ such that $\nu_{\varphi, 1} \neq 0$ on a set of positive $\mathcal{H}^{2}$-measure, or such that $S_{\varphi}$ is contained in a piecewise $\mathrm{C}^{1}$-hypersurface whose projection on the $x_{2} x_{3}$-coordinate plane is piecewise $\mathrm{C}^{1}$ in $\omega$.

Theorem 5.2 (homogenization of the MBS with nonzero cord-angle). Let $\Omega, \Omega_{\varepsilon_{k}}^{\gamma}$, $\Gamma_{C, \varepsilon_{k}}^{\gamma}$ be given like in Definition of Geometry 3.2. Suppose that the elastic energy density $W$ satisfies $(W 1), \ldots,(W 4)$ and the surface energy density $\theta$ obeys $(\theta 1), \ldots,(\theta 3)$. Define the homogenized total energy

$$
\begin{aligned}
& \mathcal{F}_{\text {Hom }}^{\gamma}: \operatorname{SBV}^{p}\left(\Omega ; \mathbb{R}^{3}\right) \cap \operatorname{Kin}(\Omega ; \operatorname{Box}) \rightarrow[0, \infty], \\
& \qquad \mathcal{F}_{\text {Hom }}^{\gamma}(\varphi):= \begin{cases}\int_{\Omega} W(\nabla \varphi) \mathrm{d} x+\int_{S_{\varphi}} \theta\left(\left|\varphi^{+}-\varphi^{-}\right|\right) \mathrm{d} \mathcal{H}^{2} \\
\infty & \text { if } \nu_{\varphi, 1}=\nu_{\varphi, 2}=0 \mathcal{H}^{2} \text {-a.e. on } S_{\varphi},\end{cases}
\end{aligned}
$$

Then for the sequence $\left(\mathcal{F}_{\varepsilon_{k}}^{\gamma}\right)_{k}$ and the homogenized total energy $\mathcal{F}_{\text {Hom }}^{\gamma}$ there holds

$$
\left(\Gamma-\lim _{k \rightarrow \infty} \mathcal{F}_{\varepsilon_{k}}^{\gamma}\right)(\varphi)=\mathcal{F}_{\mathrm{Hom}}^{\gamma}(\varphi) \quad \text { w.r.t. strong } \mathrm{L}^{1}\left(\Omega ; \mathbb{R}^{3}\right) \text {-convergence }
$$

in at least all $\varphi \in \operatorname{SBV}^{p}\left(\Omega ; \mathbb{R}^{3}\right) \cap \operatorname{Kin}(\Omega ; \mathbf{B o x})$ such that $S_{\varphi}$ is contained in a piecewise $\mathrm{C}^{1}$-hypersurface.

Remark 5.1. The reader might turn to [24], Sections 4.4.1 and 4.5.1, for a heuristic derivation of these homogenization limits. 
Remark 5.2. Both the homogenized total energies $\mathcal{F}_{\text {Hom }}^{0}$ and $\mathcal{F}_{\text {Hom }}^{\gamma}$ are sequentially lower semicontinuous w.r.t. strong $\mathrm{L}^{1}\left(\Omega ; \mathbb{R}^{3}\right)$-convergence and admit minimizers. This can easily be seen by applying Proposition 4.5 : in the case of $\mathcal{F}_{\text {Hom }}^{0}$ we set $M:=\left\{\varphi \in \operatorname{SBV}^{p}\left(\Omega ; \mathbb{R}^{3}\right): \nu_{\varphi, 1}=0 \mathcal{H}^{2}\right.$-a.e. on $\left.S_{\varphi}\right\}$ which is closed w.r.t. weak convergence in $\operatorname{SBV}^{p}\left(\Omega ; \mathbb{R}^{3}\right)$ by Proposition 4.6, furthermore $\phi(v):=\left|v_{1}\right|+\left|v_{2}\right|+\left|v_{3}\right|$ and $F:=0$; in the case of $\mathcal{F}_{\text {Hom }}^{\gamma}$ the assertion follows similarly with $M:=\left\{\varphi \in \operatorname{SBV}^{p}\left(\Omega ; \mathbb{R}^{3}\right): \nu_{\varphi, 1}=\nu_{\varphi, 2}=0 \mathcal{H}^{2}\right.$-a.e. on $\left.S_{\varphi}\right\}, \phi(v):=|v|$ and $F:=0$.

We will prove the homogenization results Theorems 5.1 and 5.2 separately in the respective subsections to come. Of particular importance - for the construction of recovery sequences - will be the concept of predeformations.

\section{Proof of the homogenization results: Zero cord-angle}

Suppose the assumptions of Theorem 5.1 to be valid throughout the present subsection.

Lemma 5.3 (Г-lim inf-inequality). Let $\varphi$ and $\left(\varphi_{k}\right)_{k}$ be in $\operatorname{SBV}^{p}\left(\Omega ; \mathbb{R}^{3}\right) \cap \operatorname{Kin}(\Omega ; \operatorname{Box})$ such that $\varphi_{k} \rightarrow \varphi$ in $\mathrm{L}^{1}\left(\Omega ; \mathbb{R}^{3}\right)$. Then

$$
\mathcal{F}_{\text {Hom }}^{0}(\varphi) \leq \liminf _{k \rightarrow \infty} \mathcal{F}_{\varepsilon_{k}}^{0}\left(\varphi_{k}\right) .
$$

Proof. Without loss of generality we assume $\infty>\liminf \mathcal{F}_{k} \mathcal{F}_{\varepsilon_{k}}^{0}\left(\varphi_{k}\right)=\lim _{m} \mathcal{F}_{\varepsilon_{k(m)}}^{0}\left(\varphi_{k(m)}\right)$. Thus for all $m \in \mathbb{N}$ we have $\varphi_{k(m)} \in \mathrm{W}^{1, p}\left(\Omega_{\varepsilon_{k(m)}}^{0} ; \mathbb{R}^{3}\right)$. Since $\Omega_{\varepsilon_{k(m)}}^{0}=\Omega \backslash \Gamma_{C, \varepsilon_{k(m)}}^{0}$ and $\Gamma_{C, \varepsilon_{k(m)}}^{0}$ is piecewise $\mathrm{C}^{1}$, Remark 4.3 implies $\nu_{\varphi_{k(m)}} \in\left\{ \pm e_{2}, \pm e_{3}\right\}$ in $\mathcal{H}^{2}$-a.e. point of $S_{\varphi_{k(m)}}$. In particular, we have

$$
\nu_{\varphi_{k(m)}, 1}=0 \quad \text { and } \quad\left\|\nu_{\varphi_{k(m)}}\right\|_{1}=1 \quad \mathcal{H}^{2} \text {-a.e., }
$$

wherein $\|v\|_{1}:=\left|v_{1}\right|+\left|v_{2}\right|+\left|v_{3}\right|$ denotes the 1-norm in $\mathbb{R}^{3} \ni v$.

The set $M:=\left\{\varphi \in \operatorname{SBV}^{p}\left(\Omega ; \mathbb{R}^{3}\right): \nu_{\varphi, 1}=0 \mathcal{H}^{2}\right.$-a.e. on $\left.S_{\varphi}\right\}$ is closed w.r.t. weak convergence in $\operatorname{SBV}^{p}\left(\Omega ; \mathbb{R}^{3}\right)$ by Proposition 4.6. With the help of Proposition 4.5 (wherein we set $F:=0$ ) we infer the sequential lower semicontinuity of the functional

$$
\begin{aligned}
\tilde{\mathcal{F}}: \operatorname{SBV}^{p}\left(\Omega ; \mathbb{R}^{3}\right) \cap \operatorname{Kin}(\Omega ; \mathbf{B o x}) & \rightarrow[0, \infty] \\
\tilde{\mathcal{F}}(\psi) & :=\left\{\begin{array}{ll}
\int_{\Omega} W(\nabla \psi) \mathrm{d} x+\int_{S_{\varphi}}\left\|\nu_{\psi}\right\|_{1} \theta\left(\left|\psi^{+}-\psi^{-}\right|\right) \mathrm{d} \mathcal{H}^{2} \\
\infty & \text { if } \psi \in M, \\
\text { else }
\end{array}\right\}=\mathcal{F}_{\text {Hom }}^{0}(\psi)
\end{aligned}
$$

w.r.t. strong $\mathrm{L}^{1}\left(\Omega ; \mathbb{R}^{3}\right)$-convergence. Consequently $\mathcal{F}_{\text {Hom }}^{0}(\varphi)=\tilde{\mathcal{F}}(\varphi) \leq \lim \inf _{m} \tilde{\mathcal{F}}\left(\varphi_{k(m)}\right)$. Taking into account the definition of $M$, equation (5.4) and the fact that $\varphi_{k(m)} \in \mathrm{W}^{1, p}\left(\Omega_{\varepsilon_{k(m)}}^{0} ; \mathbb{R}^{3}\right)$, it is easily seen that $\tilde{\mathcal{F}}\left(\varphi_{k(m)}\right)=$ $\mathcal{F}_{\varepsilon_{k(m)}}^{0}\left(\varphi_{k(m)}\right)$ for all $m \in \mathbb{N}$.

The proof of the nontrivial part of the -lim sup-statement in Theorem 5.1 is a lot more difficult and requires considerable technical effort. The following lemma marks the first step.

Lemma 5.4. Let $\varphi \in \operatorname{SBV}^{p}\left(\Omega ; \mathbb{R}^{3}\right) \cap \operatorname{Kin}(\Omega ;$ Box $)$ be such that $S_{\varphi}$ is contained in a piecewise $\mathrm{C}^{1}$-hypersurface $S$ whose projection on the $x_{2} x_{3}$-coordinate plane is piecewise $\mathrm{C}^{1}$ in $\omega$. Furthermore assume $\mathcal{F}_{\mathrm{Hom}}^{0}(\varphi)<\infty$. Then there is a sequence $\left(\varphi_{k}\right)_{k}$ in $\mathcal{V}^{p}\left(\Omega ; \mathbb{R}^{3}\right) \cap \operatorname{Kin}(\Omega ; \mathbf{B o x})$ such that

$$
\varphi_{k} \rightarrow \varphi \quad \text { in } \mathrm{L}^{1}\left(\Omega ; \mathbb{R}^{3}\right) \quad \text { and } \quad \lim _{k \rightarrow \infty} \mathcal{F}_{\mathrm{Hom}}^{0}\left(\varphi_{k}\right)=\mathcal{F}_{\mathrm{Hom}}^{0}(\varphi) .
$$

By Proposition 4.1 and Lemma 5.4 it would suffice to show the $\Gamma$-limsup-inequality for $\varphi \in \mathcal{V}^{p}\left(\Omega ; \mathbb{R}^{3}\right) \cap$ $\operatorname{Kin}(\Omega ; \operatorname{Box})$ with $\mathcal{F}_{\text {Hom }}^{0}(\varphi)<\infty$ only. However, in the polyhedral discontinuity set of such $\varphi$ still arbitrarily 
many faces can meet. Other than in the case of $\varphi_{k} \in \mathrm{W}^{1, p}\left(\Omega_{\varepsilon_{k}}^{0} ; \mathbb{R}^{3}\right) \cap \operatorname{Kin}(\Omega ; \mathbf{B o x})$ where only four faces can meet because the discontinuity set $S_{\varphi_{k}}$ is contained in $\Gamma_{C, \varepsilon_{k}}^{0} \subseteq[0, \ell] \times \varepsilon_{k} \mathcal{G}$. Here $\mathcal{G}$ is the two-dimensional grid

$$
\mathcal{G}:=\left\{x: x \in \mathbb{R}^{2}, x_{1} \in \mathbb{Z} \text { or } x_{2} \in \mathbb{Z}\right\}
$$

But elements of a recovery sequence $\left(\varphi_{k}\right)_{k}$ for $\varphi$ clearly must satisfy $\varphi_{k} \in \mathrm{W}^{1, p}\left(\Omega_{\varepsilon_{k}}^{0} ; \mathbb{R}^{3}\right) \cap \operatorname{Kin}(\Omega ;$ Box $)$. The difficulty of too many faces meeting is avoided by the next lemma.

Lemma 5.5. Let $\varphi \in \mathcal{V}^{p}\left(\Omega ; \mathbb{R}^{3}\right) \cap \operatorname{Kin}(\Omega ;$ Box $)$ be such that $\mathcal{F}_{\text {Hom }}^{0}(\varphi)<\infty$. Then there are a subsequence $\left(\varepsilon_{k(m)}\right)_{m}$ and for every $m \in \mathbb{N}$ a deformation $\varphi_{m} \in \mathcal{V}^{p}\left(\Omega ; \mathbb{R}^{3}\right) \cap \operatorname{Kin}(\Omega ; \operatorname{Box})$ with $S_{\varphi_{m}} \subseteq[0, \ell] \times P_{\omega, m}$ for a polyhedral set $P_{\omega, m} \subseteq \mathbb{R}^{2}$, such that:

(i) $\operatorname{Knot}\left(P_{\omega, m}\right) \cap \omega \subseteq \varepsilon_{k(m)} \mathbb{Z}^{2}$,

(ii) for all $L \in \operatorname{Face}\left(P_{\omega, m}\right)$ with $\operatorname{dist}(L, \partial \omega)=0$ there holds $L \subseteq \varepsilon_{k(m)} \mathcal{G}$,

(iii) for every $K \in \operatorname{Knot}\left(P_{\omega, m}\right) \cap \omega$ there are at most four elements $L_{1}, \ldots, L_{4} \in \operatorname{Face}\left(P_{\omega, m}\right)$ containing $K$ and there holds either:

for every $I \in\left\{\left[0, \frac{\pi}{2}\right),\left[\frac{\pi}{2}, \pi\right),\left[\pi, \frac{3 \pi}{2}\right),\left[\frac{3 \pi}{2}, 2 \pi\right)\right\}$ there is at maximum one $i$ such that angle between the half-line $K+\mathbb{R}_{>}[1,0]^{T}$ and $L_{i}$ is in $I$

or

for every $I \in\left\{\left(0, \frac{\pi}{2}\right],\left(\frac{\pi}{2}, \pi\right],\left(\pi, \frac{3 \pi}{2}\right],\left(\frac{3 \pi}{2}, 2 \pi\right]\right\}$ there is at maximum one $i$ such that angle between the half-line $K+\mathbb{R}_{>}[1,0]^{T}$ and $L_{i}$ is in $I$.

Moreover, it is

$$
\varphi_{m} \rightarrow \varphi \quad \text { in } \mathrm{L}^{1}\left(\Omega ; \mathbb{R}^{3}\right) \quad \text { and } \quad \lim _{m \rightarrow \infty} \mathcal{F}_{\mathrm{Hom}}^{0}\left(\varphi_{m}\right)=\mathcal{F}_{\mathrm{Hom}}^{0}(\varphi) .
$$

Comments on the proofs of these two lemmas will follow later on. We now state the construction of recovery sequences for the particular deformations of Lemma 5.5.

Lemma 5.6 (Г-limsup-inequality). Let $\psi \in \mathcal{V}^{p}\left(\Omega ; \mathbb{R}^{3}\right) \cap \operatorname{Kin}(\Omega ; \operatorname{Box})$ with $\mathcal{F}_{\mathrm{Hom}}^{0}(\psi)<\infty$ and $P_{\omega} \subseteq \mathbb{R}^{2}$ be polyhedral with $S_{\psi} \subseteq[0, \ell] \times P_{\omega}=: P$. Assume that $P_{\omega}$ satisfies the assumptions (i), (ii) and (iii) from Lemma 5.5 (where some $\varepsilon_{m}$ replaces $\varepsilon_{k(m)}$ in (i) and (ii)). Then there exists a sequence $\left(\psi_{k}\right)_{k}$ with $\psi_{k} \in$ $\mathrm{W}^{1, p}\left(\Omega_{\varepsilon_{k}}^{0} ; \mathbb{R}^{3}\right) \cap \operatorname{Kin}(\Omega ;$ Box $)$ satisfying

$$
\psi_{k} \rightarrow \psi \quad \text { in } \mathrm{L}^{1}\left(\Omega ; \mathbb{R}^{3}\right) \quad \text { and } \quad \lim _{k \rightarrow \infty} \mathcal{F}_{\varepsilon_{k}}^{0}\left(\psi_{k}\right)=\mathcal{F}_{\mathrm{Hom}}^{0}(\psi)
$$

Proof. The outline of the proof is as follows. First, for all but finitely many $k$ we construct a predeformation $\Phi_{k}: \Omega \rightarrow \Omega$ that:

- differs from the identity mapping only in an $\varepsilon_{k}$-neighborhood $\mathcal{T}_{k}$ of the polyhedral set $P$,

- is such that $\Phi_{k}^{-1}(P) \subseteq[0, \ell] \times \varepsilon_{k} \mathcal{G}$,

- satisfies for some positive constants $c_{1}, c_{2}, c_{3}$ independent of $k$ the estimate

$$
c_{1} \leq \operatorname{det} \mathrm{D} \Phi_{k} \leq c_{2}, \quad\left|\mathrm{D} \Phi_{k}\right| \leq c_{3}
$$

uniformly on $\Omega$. 
Like in Example 4.1, we define the desired sequence $\psi_{k}$ by composition of $\psi$ with the predeformations $\Phi_{k}$, i.e. $\psi_{k}:=\psi \circ \Phi_{k}$, and show $\psi_{k} \in \mathrm{W}^{1, p}\left(\Omega_{\varepsilon_{k}}^{0} ; \mathbb{R}^{3}\right) \cap \operatorname{Kin}(\Omega ;$ Box $)$. In order to prove $\psi_{k} \rightarrow \psi$ in $\mathrm{L}^{1}\left(\Omega ; \mathbb{R}^{3}\right)$ and

$$
\lim _{k \rightarrow \infty} \int_{\Omega} W\left(\nabla \psi_{k}\right) \mathrm{d} x=\int_{\Omega} W(\nabla \psi) \mathrm{d} x
$$

it suffices to exploit the uniform estimates on $\mathrm{D} \Phi_{k}, \operatorname{vol} \mathcal{T}_{k} \rightarrow 0$, the chain rule formula for predeformations (see Prop. 4.8) and (W4). As concerns convergence of the surface energies

$$
\lim _{k \rightarrow \infty} \int_{S_{\psi_{k}}} \theta\left(\left|\psi_{k}^{+}-\psi_{k}^{-}\right|\right) \mathrm{d} \mathcal{H}^{2}=\int_{S_{\psi}} \phi\left(\nu_{\psi}\right) \theta\left(\left|\psi^{+}-\psi^{-}\right|\right) \mathrm{d} \mathcal{H}^{2}
$$

some additional effort is required. The convergences (5.6) and (5.7) then imply $\lim _{k} \mathcal{F}_{\varepsilon_{k}}^{0}\left(\psi_{k}\right)=\mathcal{F}_{\text {Hom }}^{0}(\psi)$. In order to improve readability we will split the proof into several steps.

Step 1. Due to the particular form $P=[0, \ell] \times P_{\omega}$ it suffices to construct a predeformation $\Phi_{\omega, k}: \omega \rightarrow \omega$ such that $\Phi_{\omega, k}^{-1}\left(P_{\omega}\right) \subseteq \varepsilon_{k} \mathcal{G}$. We obtain the desired predeformation $\Phi_{k}$ by setting

$$
\Phi_{k}(x):=\left[\begin{array}{c}
x_{1} \\
\Phi_{\omega, k}\left(x_{2}, x_{3}\right)
\end{array}\right]
$$

for $x \in \Omega=(0, \ell) \times \omega$. This yields in particular $\Phi_{k}^{-1}(P) \subseteq[0, \ell] \times \varepsilon_{k} \mathcal{G}$. Moreover, suppose that we can construct $\Phi_{\omega, k}$ such that it differs from the identity mapping only in an $\varepsilon_{k}$-neighborhood $\mathcal{T}_{\omega, k}$ of $P_{\omega}$ and admits an estimate like (5.5) uniformly on $\omega$ with constants independent of $k$. Then also $\Phi_{k}$ differs from the identity mapping only in the $\varepsilon_{k}$-neighborhood $\mathcal{T}_{k}:=(0, \ell) \times \mathcal{T}_{\omega, k}$ of $P$ and obeys estimate (5.5) uniformly on $\Omega$ with constants independent of $k$. The construction of $\Phi_{\omega, k}$ is carried out in Steps 2, 3 and 4.

Step 2. By assumption (i) of the lemma we have $\operatorname{Knot}\left(P_{\omega}\right) \cap \omega \subseteq \varepsilon_{m} \mathbb{Z}^{2}$. Since the sequence $\left(\varepsilon_{k}\right)_{k}$ is refining, i.e. $\frac{\varepsilon_{k}}{\varepsilon_{k+1}} \in \mathbb{N}$ for all $k \in \mathbb{N}$, it follows that $\operatorname{Knot}\left(P_{\omega}\right) \cap \omega \subseteq \varepsilon_{k} \mathbb{Z}^{2}$ for all $k \geq m$. From now on we assume $k \geq m$. Let $K_{1}, K_{2} \in \operatorname{Knot}\left(P_{\omega}\right) \cap \omega$ be such that $L:=\operatorname{conv}\left\{K_{1}, K_{2}\right\} \in \operatorname{Face}\left(P_{\omega}\right)$ does not contain any other knot of $P_{\omega}$ and $\operatorname{dist}(L, \partial \omega)>0$. Let $\alpha_{i}$ denote the angle between the half-line $K_{i}+\mathbb{R}_{>} e_{1}$ and $L, i \in\{1,2\}$; obviously $\alpha_{2}=\left(\pi+\alpha_{1}\right) \bmod 2 \pi$. Regarding assumption (iii), we may assume without loss of generality that $\alpha_{1}$ is positive and in $\left[0, \frac{\pi}{2}\right)$. Moreover there shall be no other element in $\operatorname{Face}\left(P_{\omega}\right)$ that contains $K_{1}$ and encloses an angle in $\left[0, \frac{\pi}{2}\right)$ with $K_{1}+\mathbb{R}_{>} e_{1}$. For $\alpha_{2} \in\left(\pi, \frac{3 \pi}{2}\right)$ we assume that there is no other element in Face $\left(P_{\omega}\right)$ containing $K_{2}$ and enclosing an angle in $\left[\pi, \frac{3 \pi}{2}\right)$ with $K_{2}+\mathbb{R}_{>} e_{1}$. All other possible cases for $\alpha_{1}, \alpha_{2}$ can be treated analogously. In order to reduce the number of indices involved, we further assume without restriction $K_{1}=0$ and drop the index in $K_{2}$ and also in $\alpha_{1}$ (thus $L=\operatorname{conv}\{0, K\}$ ).

Now we construct a neighborhood $\mathcal{T}_{\omega, k, L}$ of $L$ composed of two closed trapezoids like shown in Figure 4 (left). By property (iii) of $P_{\omega}$ and the geometry of $P_{\omega}$ in 0 and $K$ we find angles $\beta_{1}, \ldots, \beta_{4} \in\left(0, \frac{\pi}{2}\right)$ and a sufficiently large fixed number $M \in \mathbb{N}$ depending on $\beta_{1}, \ldots, \beta_{4}$ and $L$ only such that:

- the closed set $\mathcal{T}_{\omega, k, L}$ does not intersect any other element of Face $\left(P_{\omega}\right)$, except in 0 or in $K$,

- the line segments $0+\varepsilon_{k} \operatorname{conv}\left\{0, K_{, 1} e_{1}\right\}$ and $K-\varepsilon_{k} \operatorname{conv}\left\{0, K_{, 1} e_{1}\right\}$ are contained in the interior of $\mathcal{T}_{\omega, k, L}$.

Therein, $K_{, 1}, K_{, 2} \in \mathbb{Z}$ are such that $\varepsilon_{m} \mathbb{Z}^{2} \ni K=\varepsilon_{m}\left[K_{, 1}, K_{, 2}\right]^{T}$. For $\varepsilon_{k}$ sufficiently small $\mathcal{T}_{\omega, k, L}$ becomes a subset of $\omega$. 

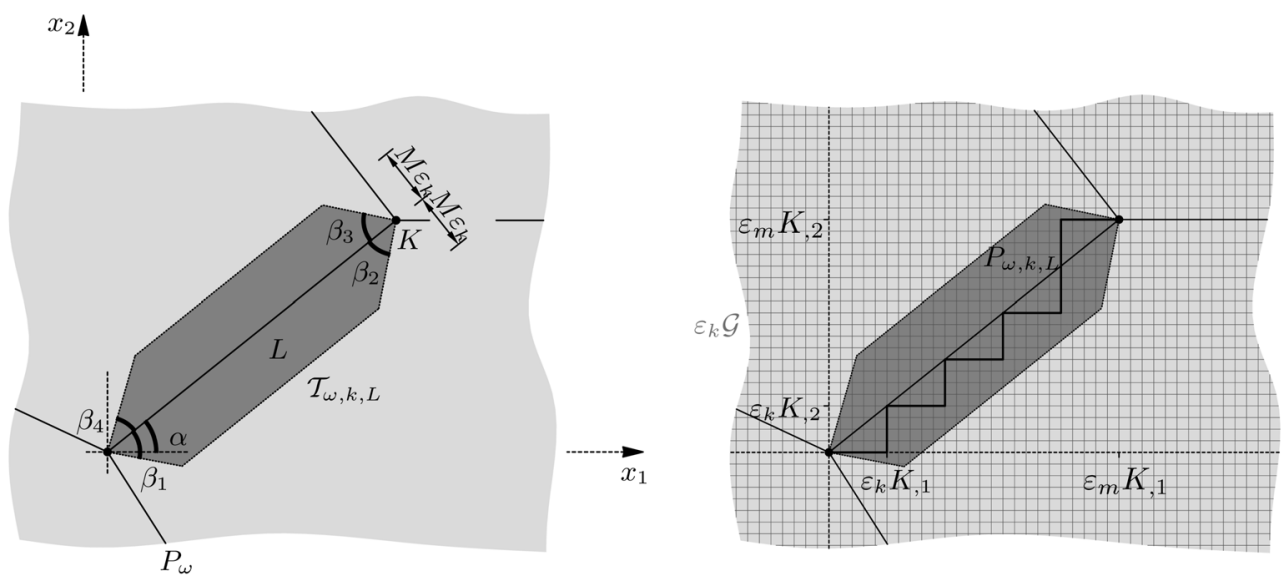

Figure 4. The trapezoidal neighborhood $\mathcal{T}_{\omega, k, L}$ (left) and the polygon $P_{\omega, k, L}$ (right).

Step 3. Thanks to the refinement property $\frac{\varepsilon_{m}}{\varepsilon_{k}} \in \mathbb{N}$ and $K=\varepsilon_{m}\left[K_{, 1}, K_{, 2}\right]^{T}, K_{, 1}, K_{, 2} \in \mathbb{Z}$, we can write

$$
\begin{aligned}
K= & \left(\varepsilon_{k}\left[\begin{array}{c}
K_{, 1} \\
0
\end{array}\right]+\varepsilon_{k}\left[\begin{array}{c}
0 \\
K_{, 2}
\end{array}\right]\right) \\
& +\ldots \\
& +\left(\varepsilon_{k}\left[\begin{array}{c}
K_{, 1} \\
0
\end{array}\right]+\varepsilon_{k}\left[\begin{array}{c}
0 \\
K_{, 2}
\end{array}\right]\right) \\
& +\left(\varepsilon_{k}\left[\begin{array}{c}
0 \\
K_{, 2}
\end{array}\right]+\varepsilon_{k}\left[\begin{array}{c}
K_{, 1} \\
0
\end{array}\right]\right) .
\end{aligned}
$$

With this representation at hand we can easily construct a rectangular polygon $P_{\omega, k, L}$ which connects 0 and $K$, is "close" to $L$ and contained in $\varepsilon_{k} \mathcal{G}$, see Figure 4 (right). By the second property of $\mathcal{T}_{\omega, k, L}$ stated in Step 1 it follows $P_{\omega, k, L} \subseteq \mathcal{T}_{\omega, k, L}$. Now we define a predeformation $\Phi_{\omega, k, L}: \mathcal{T}_{\omega, k, L} \rightarrow \mathcal{T}_{\omega, k, L}$ that:

- maps $P_{\omega, k, L}$ onto $L$,

- equals the identity mapping on $\partial \mathcal{T}_{\omega, k, L}$,

- admits an estimate like (5.5) for $\mathrm{D} \Phi_{\omega, k, L}$ uniformly on $\mathcal{T}_{\omega, k, L}$ with constants independent of $k$.

Indeed we can choose $\Phi_{\omega, k, L}$ as piecewise affine. First, we divide $\mathcal{T}_{\omega, k, L}$ into $\kappa:=2 \frac{\varepsilon_{m}}{\varepsilon_{k}}$ disjoint stripes $\mathcal{S}_{1}, \ldots, \mathcal{S}_{\kappa}$ by taking the perpendiculars on $L$ in every kink of $P_{\omega, k, L}$, see Figure 5 (left). Each of the stripes $\mathcal{S}_{i}$ is divided by $P_{\omega, k, L}$ into an upper part $\mathcal{S}_{i}^{+}$and a lower part $\mathcal{S}_{i}^{-}$. The predeformation $\Phi_{\omega, k, L}$ is on each $\mathcal{S}_{i}^{ \pm}$defined as the piecewise affine function which "moves" the outer kink of $P_{\omega, k, L}$ onto $L$ along the perpendicular (see Fig. 5 (right); if necessary, the reader might consult [24], Proof of Lem. 4.5 for details). It is an easy exercise to show that $\Phi_{\omega, k, L}$ defined in this way satisfies an estimate like (5.5) uniformly on $\mathcal{T}_{\omega, k, L}$ with constants $c_{1}, c_{2}, c_{3}$ depending only on $M, L$ and $\beta_{1}, \ldots, \beta_{4}$. Clearly, $\Phi_{\omega, k, L}$ is a predeformation.

Step 4. The construction of $\Phi_{\omega, k, L}$ over $L \in \operatorname{Face}\left(P_{\omega}\right)$ does not interfere with any other element of Face $\left(P_{\omega}\right)$. Thus, we can assume that $\Phi_{\omega, k, L}$ is constructed analogously for all elements $L \in \operatorname{Face}\left(P_{\omega}\right)$ satisfying $\operatorname{dist}(L, \partial \omega)>0$ and being contained in $\omega$. Note that any $L \in \operatorname{Face}\left(P_{\omega}\right)$ with $\operatorname{dist}(L, \partial \omega)=0$ is by assumption (ii) 

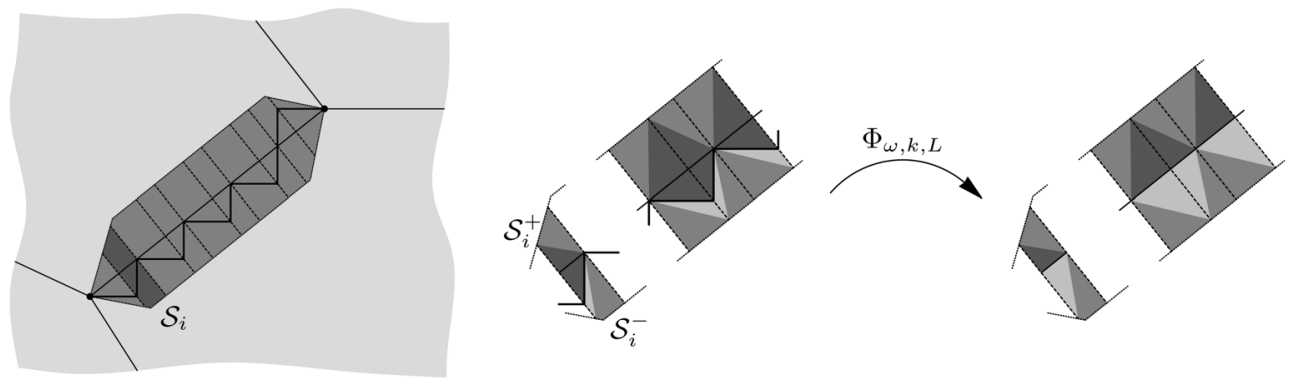

Figure 5. The stripes $\mathcal{S}_{i}$ in $\mathcal{T}_{\omega, k, L}$ (left) and the construction of $\Phi_{\omega, k, L}$ (right).

already subset of $\varepsilon_{m} \mathcal{G}$ and consequently subset of $\varepsilon_{k} \mathcal{G}$ for any $k \geq m$. On

$$
\mathcal{T}_{\omega, k}:=\bigcup\left\{\mathcal{T}_{\omega, k, L}: L \in \operatorname{Face}\left(P_{\omega}\right), \operatorname{dist}(L, \partial \omega)>0 \text { and } L \subseteq \omega\right\}
$$

we define a predeformation $\Phi_{\omega, k}$ by $\left.\Phi_{\omega, k}\right|_{\mathcal{T}_{\omega, k, L}}:=\Phi_{\omega, k, L}$. Since $\Phi_{\omega, k, L}$ maps every $\mathcal{T}_{\omega, k, L}$ onto itself and equals the identity on $\partial \mathcal{T}_{\omega, k, L}$, we can extend $\Phi_{\omega, k}$ to the whole $\mathbb{R}^{2}$ by the identity. In particular, $\Phi_{\omega, k}$ maps $\omega$ onto $\omega$ as $\mathcal{T}_{\omega, k}$ is compactly contained in $\omega$. Moreover, $\Phi_{\omega, k}$ satisfies an estimate like (5.5) uniformly on $\mathbb{R}^{2}$ with constants independent of $k$. With the help of $\Phi_{\omega, k}$ we now set $P_{\omega, k}:=\Phi_{\omega, k}^{-1}\left(P_{\omega}\right)$; by construction of $\Phi_{\omega, k}$, the intersection of the polyhedral set $P_{\omega, k}$ with $\omega$ is a subset of $\varepsilon_{k} \mathcal{G}$.

Step 5. Like explained in Step 1, we obtain the desired predeformation $\Phi_{k}: \Omega \rightarrow \Omega$ by extending $\Phi_{\omega, k}$ according to (5.8). Indeed, $\Phi_{k}$ differs from the identity mapping only on the $\varepsilon_{k}$-neighborhood $\mathcal{T}_{k}:=(0, \ell) \times \mathcal{T}_{\omega, k}$ of $P$. It also satisfies estimate (5.5) uniformly on $\Omega$ with constants independent of $k$. But in particular we have $\Phi_{k}^{-1}(P) \subseteq[0, \ell] \times P_{\omega, k}$ which in turn is by construction subset of $[0, \ell] \times \varepsilon_{k} \mathcal{G}$.

We are now in a position to define the sequence $\left(\psi_{k}\right)_{k}$ claimed in the lemma. To this end, we define for sufficiently large $k$ the deformation $\psi_{k}:=\psi \circ \Phi_{k}$ which is by Proposition 4.8 an element of $\operatorname{SBV}^{p}\left(\Omega ; \mathbb{R}^{3}\right) \cap$ $\operatorname{Kin}(\Omega$; Box $)$. From the same Proposition 4.8 we infer $S_{\psi_{k}}=\Phi_{k}^{-1}\left(S_{\psi}\right)$, while in turn $\Phi_{k}^{-1}\left(S_{\psi}\right) \subseteq \Phi_{k}^{-1}(P) \subseteq$ $[0, \ell] \times \varepsilon_{k} \mathcal{G}$. Hence, we can interpret $\psi_{k}$ as a Sobolev-function

$$
\psi_{k} \in \mathrm{W}^{1, p}\left(\Omega \backslash\left([0, \ell] \times \varepsilon_{k} \mathcal{G}\right) ; \mathbb{R}^{3}\right)=\mathrm{W}^{1, p}\left(\Omega_{\varepsilon_{k}}^{0} ; \mathbb{R}^{3}\right) .
$$

In order to prove $\psi_{k} \rightarrow \psi$ in $\mathrm{L}^{1}\left(\Omega ; \mathbb{R}^{3}\right)$ we first observe that $\psi_{k} \equiv \psi$ on $\Omega \backslash \mathcal{T}_{k}$ since $\Phi_{k}$ equals the identity on $\Omega \backslash \mathcal{T}_{k}$. The $\mathrm{L}^{1}$-convergence then follows from the uniform estimate (5.5) and the fact that the volume of $\mathcal{T}_{k}=\Phi_{k}\left(\mathcal{T}_{k}\right)$ vanishes. Similarly, due to $\psi_{k} \equiv \psi$ on $\Omega \backslash \mathcal{T}_{k}$ we can write

$$
\int_{\Omega} W\left(\nabla \psi_{k}\right) \mathrm{d} x=\int_{\Omega \backslash \mathcal{T}_{k}} W(\nabla \psi) \mathrm{d} x+\int_{\mathcal{T}_{k}} W\left(\nabla \psi_{k}\right) \mathrm{d} x .
$$

The first term on the right-hand side of (5.9) obviously converges to $\int_{\Omega} W(\nabla \psi) \mathrm{d} x$ as vol $\mathcal{T}_{k} \rightarrow 0$. As concerns the second term, using (W1), Proposition 4.8 and (W4) we write

$$
\begin{aligned}
\int_{\mathcal{T}_{k}} W\left(\nabla \psi_{k}\right) \mathrm{d} x & =\int_{\mathcal{T}_{k}} \mathrm{~W}\left(\left(\nabla \psi \circ \Phi_{k}\right) \cdot \mathrm{D} \Phi_{k}, \operatorname{det}\left(\left(\nabla \psi \circ \Phi_{k}\right) \cdot \mathrm{D} \Phi_{k}\right)\right) \mathrm{d} x \\
& \leq \int_{\mathcal{T}_{k}}^{c} c_{\mathrm{W}}\left(\mathrm{D} \Phi_{k}\right)\left(\mathrm{W}\left(\nabla \psi \circ \Phi_{k}, \operatorname{det}\left(\nabla \psi \circ \Phi_{k}\right)\right)+1\right) \mathrm{d} x .
\end{aligned}
$$




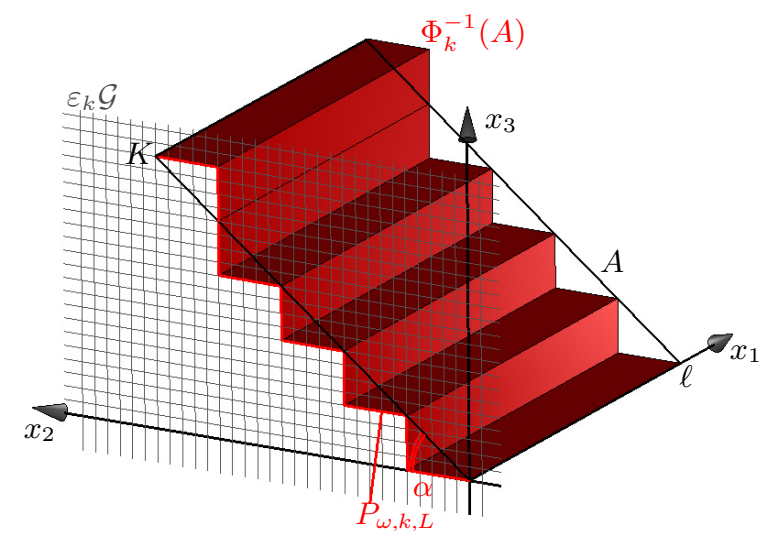

Figure 6. The face $A$ and the polyhedral set $\Phi_{k}^{-1}(A)$.

Since $c_{\mathrm{W}} \in C\left(\mathrm{M}_{>}^{3}\right)$, we deduce from (5.5) that $\left\|c_{\mathrm{W}}\left(\mathrm{D} \Phi_{k}\right)\right\|_{\mathrm{L}^{\infty}(\Omega)}$ is uniformly bounded in $k$ by some positive constant $c_{4}$. Thus, we can further estimate

$$
\int_{\mathcal{T}_{k}} W\left(\nabla \psi_{k}\right) \mathrm{d} x \leq c_{4} \int_{\mathcal{T}_{k}} \mathrm{~W}\left(\nabla \psi \circ \Phi_{k}, \operatorname{det}\left(\nabla \psi \circ \Phi_{k}\right)\right)+1 \mathrm{~d} x \leq \frac{c_{4}}{c_{1}} \int_{\mathcal{T}_{k}} \mathrm{~W}(\nabla \psi, \operatorname{det} \nabla \psi)+1 \mathrm{~d} x \rightarrow 0,
$$

where we identified $\Phi_{k}\left(\mathcal{T}_{k}\right)=\mathcal{T}_{k}$ and estimated $\operatorname{det} \mathrm{D}\left(\Phi_{k}^{-1}\right)$ from above by means of (5.5). This proves (5.6). Step 6. It remains to prove (5.7). First, from $S_{\psi_{k}} \subseteq \Phi_{k}^{-1}(P), P=[0, \ell] \times P_{\omega}$ and ( $\theta 3$ ) it follows

$$
\begin{array}{r}
\int_{S_{\psi_{k}}} \theta\left(\left|\psi_{k}^{+}-\psi_{k}^{-}\right|\right) \mathrm{d} \mathcal{H}^{2}=\sum\left\{\int_{\Phi_{k}^{-1}(A)} \theta\left(\left|\psi^{+} \circ \Phi_{k}-\psi^{-} \circ \Phi_{k}\right|\right) \mathrm{d} \mathcal{H}^{2}: A \in \operatorname{Face}(P)\right\} \\
=\sum\left\{\int_{\Phi_{k}^{-1}(A)} \theta\left(\left|\psi^{+} \circ \Phi_{k}-\psi^{-} \circ \Phi_{k}\right|\right) \mathrm{d} \mathcal{H}^{2}: A=[0, \ell] \times L, L \in \operatorname{Face}\left(P_{\omega}\right)\right\} .
\end{array}
$$

For the identity $\psi_{k}^{ \pm}=\psi^{ \pm} \circ \Phi_{k}$ we refer to Remark 4.3. Hence, it suffices to study the convergence of the finitely many integrals on the right-hand side of (5.10). Let $L$ be an element of $\operatorname{Face}\left(P_{\omega}\right), A=[0, \ell] \times L$. By construction it is $\Phi_{k}^{-1}(A)=(0, \ell) \times \Phi_{\omega, k}^{-1}(L)$.

Consider the case $\operatorname{dist}(L, \partial \omega)=0$ : since $\Phi_{\omega, k}$ equals the identity over all such $L$, we obtain

$$
\int_{\Phi_{k}^{-1}(A)} \theta\left(\left|\psi^{+} \circ \Phi_{k}-\psi^{-} \circ \Phi_{k}\right|\right) \mathrm{d} \mathcal{H}^{2}=\int_{(0, \ell) \times(L \cap \omega)} \theta\left(\left|\psi^{+}-\psi^{-}\right|\right) \mathrm{d} \mathcal{H}^{2}
$$

Moreover, according to the assumptions of the lemma $L \subseteq \varepsilon_{m} \mathcal{G}$. Therefore, a normal $\nu_{A}$ on $A=[0, \ell] \times L$ is necessarily $\pm e_{2}$ or $\pm e_{3}$ yielding $\phi\left(\nu_{A}\right)=1$. It follows

$$
\int_{\Phi_{k}^{-1}(A)} \theta\left(\left|\psi^{+} \circ \Phi_{k}-\psi^{-} \circ \Phi_{k}\right|\right) \mathrm{d} \mathcal{H}^{2}=\int_{A \cap \Omega} \phi\left(\nu_{A}\right) \theta\left(\left|\psi^{+}-\psi^{-}\right|\right) \mathrm{d} \mathcal{H}^{2}
$$

for every $A=[0, \ell] \times L, L \in \operatorname{Face}\left(P_{\omega}\right)$ with $\operatorname{dist}(L, \partial \omega)=0$.

We turn to the case $\operatorname{dist}(L, \partial \omega)>0$. Like in the Steps 2,3 and 4 we explain the procedure for $L=\operatorname{conv}\{0, K\}$, $K \in \varepsilon_{m} \mathbb{Z}^{2}$. Let $\nu_{L}=[\cos \alpha,-\sin \alpha]^{T}$ be a unit normal on $L$ and $\nu_{A}=[0, \cos \alpha,-\sin \alpha]^{T}$ be a unit normal on $A$ (see Fig. 6). By construction $\Phi_{k}^{-1}(A)=(0, \ell) \times P_{\omega, k, L}$ with $P_{\omega, k, L}=\Phi_{\omega, k}^{-1}(L) \subseteq \varepsilon_{k} \mathcal{G}$, cf. Figure 6 . We can 
parameterize the rectangular polygon $P_{\omega, k, L}$ by means of

$$
\lambda_{\omega, k}:\left(0, \mathcal{H}^{1}(L)\right) \rightarrow \mathbb{R}^{2}, \quad \lambda_{\omega, k}(s):=R_{\alpha}\left[\begin{array}{c}
s \\
\tau_{k}(s)
\end{array}\right]
$$

Herein, $R_{\alpha} \in \mathrm{SO}(2)$ is the rotation about 0 through $\alpha$ and $\tau_{k}:\left(0, \mathcal{H}^{1}(L)\right) \rightarrow \mathbb{R}$ is the zig-zag-function

$$
\tau_{k}(s):= \begin{cases}-\tan \alpha \cdot(s-b) & \text { if } s \in b+\eta_{k}\left[0,(\cos \alpha)^{2}\right], \\ \cot \alpha \cdot\left(s-\left(b+\eta_{k}\right)\right) & \text { if } s \in b+\eta_{k}\left[(\cos \alpha)^{2}, 1\right] \\ \cot \alpha \cdot\left(s-\left(\mathcal{H}^{1}(L)-\eta_{k}\right)\right) & \text { if } s \in\left(\mathcal{H}^{1}(L)-\eta_{k}\right)+\eta_{k}\left[0,1-(\cos \alpha)^{2}\right], \\ -\tan \alpha \cdot\left(s-\mathcal{H}^{1}(L)\right) & \text { if } s \in\left(\mathcal{H}^{1}(L)-\eta_{k}\right)+\eta_{k}\left[1-(\cos \alpha)^{2}, 1\right],\end{cases}
$$

$\eta_{k}:=\frac{\varepsilon_{k}}{\varepsilon_{m}} \mathcal{H}^{1}(L)$ and $b=0, \eta_{k}, 2 \eta_{k}, \ldots, \mathcal{H}^{1}(L)-2 \eta_{k}$. The corresponding parameterization of $\Phi_{k}^{-1}(A)$,

$$
\lambda_{k}(r, s):(0, \ell) \times\left(0, \mathcal{H}^{1}(L)\right) \rightarrow \mathbb{R}^{3}, \quad \lambda_{k}(r, s):=\left[\begin{array}{c}
r \\
\lambda_{\omega, k}(s)
\end{array}\right],
$$

allows us to compute

$$
\begin{aligned}
& \int_{\Phi_{k}^{-1}(A)} \theta\left(\left|\psi^{+} \circ \Phi_{k}-\psi^{-} \circ \Phi_{k}\right|\right) \mathrm{d} \mathcal{H}^{2} \\
& \quad=\int_{(0, \ell) \times\left(0, \mathcal{H}^{1}(L)\right)} \theta\left(\left|\psi^{+} \circ \Phi_{k} \circ \lambda_{k}(r, s)-\psi^{-} \circ \Phi_{k} \circ \lambda_{k}(r, s)\right|\right) \cdot \sqrt{\operatorname{det}\left(\operatorname{D}_{k}^{T}(r, s) \operatorname{D} \lambda_{k}(r, s)\right)} \mathrm{d}(r, s)
\end{aligned}
$$

It is easily verified that

$$
\operatorname{det}\left(\mathrm{D} \lambda_{k}^{T}(r, s) \mathrm{D} \lambda_{k}(r, s)\right)= \begin{cases}\frac{1}{(\cos \alpha)^{2}} & \text { if } s \in b+\eta_{k}\left[0,(\cos \alpha)^{2}\right], \\ \frac{1}{(\sin \alpha)^{2}} & \text { if } s \in b+\eta_{k}\left[(\cos \alpha)^{2}, 1\right] \\ \frac{1}{(\sin \alpha)^{2}} & \text { if } s \in\left(\mathcal{H}^{1}(L)-\eta_{k}\right)+\eta_{k}\left[0,1-(\cos \alpha)^{2}\right], \\ \frac{1}{(\cos \alpha)^{2}} & \text { if } s \in\left(\mathcal{H}^{1}(L)-\eta_{k}\right)+\eta_{k}\left[1-(\cos \alpha)^{2}, 1\right]\end{cases}
$$

where $b=0, \eta_{k}, 2 \eta_{k}, \ldots, \mathcal{H}^{1}(L)-2 \eta_{k}$. Standard two-scale arguments (see e.g. [18], Thm. 15) yield

$$
\sqrt{\operatorname{det}\left(\mathrm{D} \lambda_{k}^{T} \mathrm{D} \lambda_{k}\right)} \rightarrow|\cos \alpha|+|\sin \alpha|=\phi\left(\nu_{A}\right) \quad \text { in } \mathrm{L}^{2}\left((0, \ell) \times\left(0, \mathcal{H}^{1}(L)\right)\right) \text {. }
$$

By construction we have

$$
\Phi_{k} \circ \lambda_{k}(r, s)=\left[\begin{array}{c}
r \\
R_{\alpha}\left[\begin{array}{l}
s \\
0
\end{array}\right]
\end{array}\right]
$$

Eventually, we insert (5.13) and (5.15) into (5.12), pass to the limit $k \rightarrow \infty$ by means of (5.14) and arrive at

$$
\begin{aligned}
& \int_{\Phi_{k}^{-1}(A)} \theta\left(\left|\psi^{+} \circ \Phi_{k}-\psi^{-} \circ \Phi_{k}\right|\right) \mathrm{d} \mathcal{H}^{2} \\
\rightarrow & \int_{(0, \ell) \times\left(0, \mathcal{H}^{1}(L)\right)} \theta\left(\mid \psi^{+} \circ\left[\begin{array}{c}
r \\
R_{\alpha}[s, 0]^{T}
\end{array}\right]-\psi^{-} \circ\left[\begin{array}{c}
r \\
R_{\alpha}[s, 0]^{T}
\end{array}\right]\right) \cdot \phi\left(\nu_{A}\right) \mathrm{d}(r, s) \\
= & \int_{A} \phi\left(\nu_{A}\right) \theta\left(\left|\psi^{+}-\psi^{-}\right|\right) \mathrm{d} \mathcal{H}^{2} .
\end{aligned}
$$


This together with (5.11) allows us to pass to the limit on the right-hand side of (5.10) and we conclude

$$
\begin{aligned}
\int_{S_{\psi_{k}}} \theta\left(\left|\psi_{k}^{+}-\psi_{k}^{-}\right|\right) \mathrm{d} \mathcal{H}^{2} & \rightarrow \sum_{\{}\left\{\int_{A} \phi\left(\nu_{A}\right) \theta\left(\left|\psi^{+}-\psi^{-}\right|\right) \mathrm{d} \mathcal{H}^{2}: A=[0, \ell] \times L, L \in \operatorname{Face}\left(P_{\omega}\right)\right\} \\
& =\int_{S_{\psi}} \phi\left(\nu_{\psi}\right) \theta\left(\left|\psi^{+}-\psi^{-}\right|\right) \mathrm{d} \mathcal{H}^{2}
\end{aligned}
$$

Thus we have (5.7) and the proof of the lemma is finished.

Remark 5.3 (on the proof of Lem. 5.4). Predeformations also play a central role in the proof of Lemma 5.4 . For a deformation $\varphi$ like in Lemma 5.4 with $S_{\varphi} \subseteq S=[0, \ell] \times S_{\omega}, S_{\omega}$ a piecewise $\mathrm{C}^{1}$-hypersurface in $\omega$, we construct predeformations $\Phi_{\omega, k}: \omega \rightarrow \omega$ that:

- differ from the identity only in an $\varepsilon_{k}$-neighborhood of $S_{\omega}$,

- map the piecewise linear interpolation of $S_{\omega}$ onto $S_{\omega}$,

- satisfy a uniform estimate like (5.5).

Defining $\Phi_{k}: \Omega \rightarrow \Omega$ like in (5.8), we obtain the desired approximation of $\varphi$ by composition $\varphi_{k}:=\varphi \circ \Phi_{k}$. See [24], Lemma 4.17, for details.

Remark 5.4 (on the proof of Lem. 5.5). While sparing the reader the technical details of the proof of Lemma 5.5, we want to present one of its vital steps that illustrates once more the usefulness of pre-deformations. Again, we refer to [24], Lemma 4.13, for details. Here we show how a deformation $\varphi \in \mathcal{V}^{p}\left(\Omega ; \mathbb{R}^{3}\right) \cap \operatorname{Kin}(\Omega ; \operatorname{Box})$ with $\mathcal{F}_{\text {Hom }}^{0}(\varphi)<\infty$ and a polyhedral discontinuity set $S_{\varphi} \subseteq[0, \ell] \times P_{\omega}=: P$ can be approximated by a sequence $\left(\psi_{j}\right)_{j}$ in $\mathcal{V}^{p}\left(\Omega ; \mathbb{R}^{3}\right) \cap \operatorname{Kin}(\Omega ;$ Box $)$ such that:

- the discontinuity set of every $\psi_{j}$ is contained in a polyhedral set $P_{j}=[0, \ell] \times P_{\omega, j}$ satisfying condition (iii) from Lemma 5.5,

- it is $\psi_{j} \rightarrow \varphi$ in $\mathrm{L}^{1}\left(\Omega ; \mathbb{R}^{3}\right)$ and $\lim _{j} \mathcal{F}_{\text {Hom }}^{0}\left(\psi_{j}\right)=\mathcal{F}_{\text {Hom }}^{0}(\varphi)$.

Again, we construct appropriate predeformations $\Phi_{j}$ and define $\psi_{j}$ by composition $\psi_{j}:=\varphi \circ \Phi_{j}$. This time however, we use the predeformation to "cut out of $\Omega$ what doesn't fit": junctions of $P$ that violate condition (iii) from Lemma 5.5 (cf. also Ex. 4.1). Consider $K \in \mathcal{K}:=\operatorname{Knot}\left(P_{\omega}\right) \cap \omega$ and an open cube $Q_{j, K}$ centered at $K$ with side length $\frac{1}{j}$. For $j$ sufficiently large, $Q_{j, K}$ separates $K$ from all other elements of $\mathcal{K}$. We may choose an open triangle $\Delta_{j, K}$ that is compactly contained in $Q_{j, K}$ and does not intersect $P_{\omega}$. Let $\Phi_{\omega, j}$ be a bijective piecewise affine function with positive Jacobian determinant mapping $Q_{j, K}$ onto $\Delta_{j, K}$ for all $K \in \mathcal{K}$. Outside the cubes, i.e. on $\omega \backslash \bigcup_{\mathcal{K}} \overline{Q_{j, K}}$, suppose $\Phi_{\omega, j}$ to be the identity mapping. Analogously to (5.8) we define

$$
\Phi_{j}:(0, \ell) \times \omega_{j, \text { sliced }} \rightarrow(0, \ell) \times \omega_{j, \text { perf }}, \quad \Phi_{j}(x):=\left[\begin{array}{c}
x_{1} \\
\Phi_{\omega, j}\left(x_{2}, x_{3}\right)
\end{array}\right]
$$

where

$$
\omega_{j \text {,sliced }}:=\omega \backslash \bigcup_{\mathcal{K}} \partial Q_{j, K} \quad \text { and } \quad \omega_{j, \text { perf }}:=\left(\omega \backslash \bigcup_{\mathcal{K}} \overline{Q_{j, K}}\right) \cup \bigcup_{\mathcal{K}} \Delta_{j, K} .
$$


Indeed, $\Phi_{j}$ is a predeformation ( $c f$. [24], Proof of Lem. 4.7). An application of Proposition 4.8 then yields $\psi_{j}:=\varphi \circ \Phi_{j} \in \operatorname{SBV}^{p}\left(\Omega ; \mathbb{R}^{3}\right) \cap \operatorname{Kin}(\Omega ; \mathbf{B o x})$ with

$$
\begin{aligned}
S_{\psi_{j}} & \subseteq \Phi_{j}^{-1}\left(S_{\varphi} \cap\left([0, \ell] \times \omega_{j, \text { perf }}\right)\right) \cup\left([0, \ell] \times \bigcup_{\mathcal{K}} \partial Q_{j, K}\right) \\
& \subseteq[0, \ell] \times\left(\left(P_{\omega} \backslash \bigcup_{\mathcal{K}} \overline{Q_{j, K}}\right) \cup \bigcup_{\mathcal{K}} \partial Q_{j, K}\right)=: P_{j} .
\end{aligned}
$$

It is easily seen that $P_{j}$ satisfies condition (iii) of Lemma 5.5. Moreover, we show in [24], Proof of Lemma 4.7, that $\Phi_{j}$ admits an estimate like (5.5) uniformly on $\Omega$ for constants independent of $j$. Then, by repeating the arguments in Step 5 of the proof of Lemma 5.6 we prove the $\mathrm{L}^{1}$-convergence of $\left(\psi_{j}\right)_{j}$ to $\varphi$ and $\lim _{j} \mathcal{F}_{\text {Hom }}^{0}\left(\psi_{j}\right)=$ $\mathcal{F}_{\text {Hom }}^{0}(\varphi)$.

\section{Proof of the homogenization results: Nonzero cord-angle}

Suppose the assumptions of Theorem 5.2 to be valid. Other than in the case of zero cord-angle it is now the r-lim inf-statement of Theorem 5.2 that turns out to be the more difficult to prove. The main ingredient is the following statement.

Lemma 5.7. Consider an arbitrary subsequence $\left(\varepsilon_{k(m)}\right)_{m}$ of $\left(\varepsilon_{k}\right)_{k}$. Let $\varphi \in \operatorname{SBV}^{p}\left(\Omega ; \mathbb{R}^{3}\right)$ be such that $S_{\varphi}$ is contained in a piecewise $\mathrm{C}^{1}$-hypersurface $S$ and $\nu_{\varphi, 1} \neq 0$ or $\nu_{\varphi, 2} \neq 0$ on a subset of $S_{\varphi}$ of positive $\mathcal{H}^{2}$-measure. Then for every sequence $\left(\varphi_{m}\right)_{m}$ such that $\varphi_{m} \in \mathrm{W}^{1, p}\left(\Omega_{\varepsilon_{k(m)}^{\gamma}}^{\gamma} ; \mathbb{R}^{3}\right)$ and $\varphi_{m} \rightarrow \varphi$ in $\mathrm{L}^{1}\left(\Omega ; \mathbb{R}^{3}\right)$ we have

$$
\liminf _{m \rightarrow \infty} \int_{\Omega}\left|\nabla \varphi_{m}\right|^{p} \mathrm{~d} x=\infty
$$

Proof. By assumption, there is $x_{0} \in S_{\varphi}$ such that $\nu_{\varphi, 1}\left(x_{0}\right) \neq 0$ or $\nu_{\varphi, 2}\left(x_{0}\right) \neq 0$; by definition $\varphi^{+}\left(x_{0}\right)-\varphi^{-}\left(x_{0}\right) \neq$ 0 . The idea of the proof is the following. Due to the nonzero cord-angle $\gamma, \nu_{\varphi}\left(x_{0}\right)$ is nonorthogonal to at least one of the beam directions in $\Omega_{\varepsilon_{k(m)}}^{\gamma}$. According to Remark 4.3, $\nu_{\varphi}=\nu_{S} \mathcal{H}^{2}$-a.e. on $S_{\varphi}$, thus $S$ is in a neighborhood of $x_{0}$ nonparallel to one of the beam directions. Consequently, the set $S$ would in this neighborhood "cut through" all those beams in $\Omega_{\varepsilon_{k(m)}}^{\gamma}$ it is nonparallel to. On the other hand, any $\varphi_{m} \in \mathrm{W}^{1, p}\left(\Omega_{\varepsilon_{k(m)}}^{\gamma} ; \mathbb{R}^{3}\right)$ can only jump across the inner contact boundary $\Gamma_{C, \varepsilon_{k(m)}}^{\gamma}$, but not in beam direction. This means that as the $\varphi_{m}$ imitate the jump of $\varphi$ across $S$, the fibres of the beams being "cut through" by $S$ are stretched enormously resulting in higher and higher values of $\int_{\Omega}\left|\nabla \varphi_{m}\right|^{p} \mathrm{~d} x$.

Let us turn to the details of the proof. According to our definition of piecewise $\mathrm{C}^{1}$-hypersurfaces there are bounded Lipschitzian domains $U_{1}, \ldots, U_{k} \subseteq \mathbb{R}^{2}, g_{i} \in \mathrm{C}^{1}\left(\overline{U_{i}}\right), Q_{i} \in \mathrm{SO}(3)$ and $b_{i} \in \mathbb{R}^{3}$ such that with

$$
S_{i}:=\left\{Q_{i}\left[\hat{\xi}, g_{i}(\hat{\xi})\right]^{T}+b_{i}: \hat{\xi} \in U_{i}\right\}
$$

there holds $S=\bigcup_{i=1}^{k} \overline{S_{i}}$. Without loss of generality we may assume that $x_{0} \in S_{1}$ (not on the "boundary" of the graph $\overline{S_{1}}$, see also Rem. 2.1). To simplify the presentation, we temporarily introduce some notation. By $v_{1}, v_{2}$ we denote the beam directions $\mathcal{D}_{\gamma}$, i.e. $v_{1}=[1,0,0]^{T}$ and $v_{2}=[\cos \gamma, \sin \gamma, 0]^{T}$. The collection of all beams in $\mathcal{D}_{\gamma}$ oriented in $v_{1}$ is

$$
\mathcal{D}_{\gamma, v_{1}}:=\bigcup\left\{\left(0, a_{2}, 2 a_{3}\right)+\mathcal{B}: a_{2}, a_{3} \in \mathbb{Z}\right\}
$$

similarly

$$
\mathcal{D}_{\gamma, v_{2}}:=R_{\gamma}\left(\bigcup\left\{\left(0, a_{2}, 2 a_{3}-1\right)+\mathcal{B}: a_{2}, a_{3} \in \mathbb{Z}\right\}\right)
$$


contains all those oriented in $v_{2}$-direction. Herein, $R_{\gamma}$ is the rotation about the $x_{3}$-axis through $\gamma$ and $\mathcal{B}=$ $\mathbb{R} \times(0,1)^{2}$. Since $\gamma \neq 0$, we can always choose $v \in\left\{v_{1}, v_{2}\right\}$ such that $\nu_{\varphi}\left(x_{0}\right) \not \perp v$. Furthermore, we may assume the coordinate frame to be translated and rotated such that the origin lies in $x_{0}$ and the vertical axis (the axis of the third component) is oriented like $v$. To distinguish this new coordinate frame from the one used so far, we refer to coordinates in the new frame as $\xi=\left(\xi_{1}, \xi_{2}, \xi_{3}\right)$ (instead of $x=\left(x_{1}, x_{2}, x_{3}\right)$ used in the original frame). For the rest of the proof, every object shall be represented in the new coordinate frame.

Since $\nu_{\varphi}(0) \not \perp v$, by the implicit function theorem and the fact that $S_{1}$ is a $\mathrm{C}^{1}$-manifold we can find an open neighborhood $U \subset \mathbb{R}^{2}$ of 0 and a $g \in \mathrm{C}^{1}(U)$ such that $g(0)=0$ and $S_{1} \cap(U \times \mathbb{R})$ is the graph of $g$. Let $r>0$ be sufficiently small such that $S_{1}$ divides $B_{r}(0)$ in two parts $B_{r}^{ \pm}$. Moreover - possibly upon choosing a smaller $r-$ one has $\varphi \in \mathrm{W}^{1, p}\left(B_{r}^{ \pm} ; \mathbb{R}^{3}\right)$. From the fact that $p>3$, the Lipschitz-regularity of $\partial B_{r}^{ \pm}$for small enough $r$ and the Sobolev-imbedding theorem we infer $\varphi \in \mathrm{C}\left(\overline{B_{r}^{ \pm}} ; \mathbb{R}^{3}\right)$. Thus, we find an open set $V \subseteq U$ containing 0 and a $h_{0}>0$ such that

$$
\left|\varphi\left((\hat{\xi}, g(\hat{\xi}))+h_{+} v\right)-\varphi\left((\hat{\xi}, g(\hat{\xi}))-h_{-} v\right)\right| \geq c_{1}
$$

for all $0<h_{ \pm} \leq h_{0}, \hat{\xi} \in V$ and a positive constant $c_{1}$.

Step 1. Consider the set $A_{m}:=\left\{\hat{\xi}: \hat{\xi} \in U,(\hat{\xi}, 0) \in \varepsilon_{k(m)} \mathcal{D}_{\gamma, v}\right\}$, then $\mathbb{1}_{A_{m}} \rightarrow \frac{1}{2}$ in $\mathrm{L}^{2}(U)$. Set $Q_{m}:=A_{m} \cap V$, then $\operatorname{vol}_{2} Q_{m}=\int_{U} \mathbb{1}_{A_{m}} \cdot \mathbb{1}_{V} \mathrm{~d} \hat{\xi} \rightarrow \frac{1}{2} \operatorname{vol}_{2} V$. Therefore, we may without loss of generality assume that for all elements of the sequence $\left(\varepsilon_{k(m)}\right)_{m}$ there holds

$$
c_{2} \operatorname{vol}_{2} V \leq \operatorname{vol}_{2} Q_{m} \leq c_{3} \operatorname{vol}_{2} V
$$

with the special choice $c_{2}=\frac{3}{8}, c_{3}=\frac{5}{8}$.

Step 2. For real numbers $a_{1}<a_{2}$ we define

$$
Z_{m}\left(a_{1}, a_{2}\right):=\left\{\left(\hat{\xi}, \xi_{3}\right): \hat{\xi} \in Q_{m}, g(\hat{\xi})+a_{1} \leq \xi_{3} \leq g(\hat{\xi})+a_{2}\right\} .
$$

Claim. There holds for $0<h_{ \pm} \leq h_{0}$

$$
\int_{Z_{m}\left(-h_{-}, h_{+}\right)}\left|\nabla \varphi_{m}\right|^{p} \mathrm{~d} \xi \geq \frac{1}{\left(h_{+}+h_{-}\right)^{p-1}} \int_{Q_{m}}\left|\varphi_{m}\left((\hat{\xi}, g(\hat{\xi}))+h_{+} v\right)-\varphi_{m}\left((\hat{\xi}, g(\hat{\xi}))-h_{-} v\right)\right|^{p} \mathrm{~d} \hat{\xi} .
$$

Proof of the claim. Let $\left(\psi_{n}\right)_{n}$ be a sequence in $\mathrm{C}^{\infty}\left(\Omega_{\varepsilon_{k(m)}}^{\gamma} ; \mathbb{R}^{3}\right)$ such that $\psi_{n} \rightarrow \varphi_{m}$ in $\mathrm{W}^{1, p}\left(\Omega_{\varepsilon_{k(m)}}^{\gamma} ; \mathbb{R}^{3}\right)$. From the Rellich-Kondrachov-imbedding theorem, the fact that $p>3$ and the regularity of the subbodies forming $\Omega_{\varepsilon_{k(m)}}^{\gamma}$ we obtain $\psi_{n} \rightarrow \varphi_{m}$ uniformly on the closure of each of the subbodies. Hence, $\psi_{n} \rightarrow \varphi_{m}$ uniformly on $\bar{\Omega}$. For $\hat{\xi} \in Q_{m}$ set $\alpha:\left(-h_{-}, h_{+}\right) \rightarrow \mathbb{R}^{3}, \alpha(t):=\psi_{n}((\hat{\xi}, g(\hat{\xi}))+t v)$. Then, since $t \mapsto(\hat{\xi}, g(\hat{\xi}))+t v$ describes a fibre in a beam of $\varepsilon_{k(m)} \mathcal{D}_{\gamma, v}$ and $\psi_{n}$ is smooth on $\Omega_{\varepsilon_{k(m)}^{\gamma}}^{\gamma}=\Omega \cap \varepsilon_{k(m)} \mathcal{D}_{\gamma}, \alpha$ is a smooth curve in $\mathbb{R}^{3}$ connecting $\psi_{n}\left((\hat{\xi}, g(\hat{\xi}))-h_{-} v\right)$ and $\psi_{n}\left((\hat{\xi}, g(\hat{\xi}))+h_{+} v\right)$. We obtain

$$
\begin{aligned}
\left|\psi_{n}\left((\hat{\xi}, g(\hat{\xi}))+h_{+} v\right)-\psi_{n}\left((\hat{\xi}, g(\hat{\xi}))-h_{-} v\right)\right| \leq \text { "length of } \alpha " & =\int_{-h_{-}}^{h_{+}}|\dot{\alpha}(t)| \mathrm{d} t \\
& =\int_{-h_{-}}^{h_{+}}\left|\mathrm{D} \psi_{n}((\hat{\xi}, g(\hat{\xi}))+t v) \cdot v\right| \mathrm{d} t .
\end{aligned}
$$

Using the change of variables $\left(\hat{\xi}, \xi_{3}\right) \mapsto\left(\hat{\xi}, g(\hat{\xi})+\xi_{3}\right)$ and Fubini's theorem we can write

$$
\int_{Z_{m}\left(-h_{-}, h_{+}\right)}\left|\mathrm{D} \psi_{n}\right|^{p} \mathrm{~d} \xi=\int_{Q_{m}} \int_{-h_{-}}^{h_{+}}\left|\mathrm{D} \psi_{n}((\hat{\xi}, g(\hat{\xi}))+t v)\right|^{p} \mathrm{~d} t \mathrm{~d} \hat{\xi}
$$


the Jacobian determinant in the change of variables being identically 1. Furthermore, recall that the matrix norm $|\cdot|$ can be estimated from below by $|M| \geq \sup _{|w|=1}|M w| \geq|M v|$. This together with (5.20) and (5.19) gives

$$
\begin{aligned}
\int_{Z_{m}\left(-h_{-}, h_{+}\right)}\left|\mathrm{D} \psi_{n}\right|^{p} \mathrm{~d} \xi & \geq \int_{Q_{m}} \int_{-h_{-}}^{h_{+}}\left|\mathrm{D} \psi_{n}((\hat{\xi}, g(\hat{\xi}))+t v) \cdot v\right|^{p} \mathrm{~d} t \mathrm{~d} \hat{\xi} \\
& \geq \int_{Q_{m}} \frac{1}{\left(h_{+}+h_{-}\right)^{p-1}}\left(\int_{-h_{-}}^{h_{+}}\left|\mathrm{D} \psi_{n}((\hat{\xi}, g(\hat{\xi}))+t v) \cdot v\right| \mathrm{d} t\right)^{p} \mathrm{~d} \hat{\xi} \\
& \geq \int_{Q_{m}} \frac{1}{\left(h_{+}+h_{-}\right)^{p-1}}\left|\psi_{n}\left((\hat{\xi}, g(\hat{\xi}))+h_{+} v\right)-\psi_{n}\left((\hat{\xi}, g(\hat{\xi}))-h_{-} v\right)\right|^{p} \mathrm{~d} \hat{\xi},
\end{aligned}
$$

wherein we made also use of Jensen's inequality. Now taking the limit $n \rightarrow \infty$ on both sides of the inequality resulting from (5.21) proves the claim.

Step 3. Choose an $\eta \leq \frac{1}{M} c_{2} \mathrm{vol}_{2} V \cdot h_{0}$, wherein $M \in \mathbb{N}$ is fixed and will be specified later. From Egorov's theorem we infer the existence of a $\Omega^{\prime} \subseteq \Omega$ such that $\operatorname{vol}\left(\Omega \backslash \Omega^{\prime}\right)<\eta$ and $\varphi_{m} \rightarrow \varphi$ uniformly on $\Omega^{\prime}$. By Step 2 we realize

$$
\begin{aligned}
\operatorname{vol}\left(Z_{m}\left(0, h_{0}\right) \cap \Omega^{\prime}\right) \geq \operatorname{vol} Z_{m}\left(0, h_{0}\right)-\operatorname{vol}\left(\Omega \backslash \Omega^{\prime}\right) & =\operatorname{vol}_{2} Q_{m} \cdot h_{0}-\operatorname{vol}\left(\Omega \backslash \Omega^{\prime}\right) \\
& \geq c_{2} \operatorname{vol}_{2} V \cdot h_{0}-\frac{1}{M} c_{2} \operatorname{vol}_{2} V \cdot h_{0}=\frac{M-1}{M} c_{2} \operatorname{vol}_{2} V \cdot h_{0} .
\end{aligned}
$$

Moreover, there is a special $H_{m,+} \in\left(0, h_{0}\right)$ such that

$$
P_{m}^{+}:=\left\{\hat{\xi}: \hat{\xi} \in Q_{m},(\hat{\xi}, g(\hat{\xi}))+H_{m,+} v \in Z_{m}\left(0, h_{0}\right) \cap \Omega^{\prime}\right\}
$$

has volume $\operatorname{vol}_{2} P_{m}^{+} \geq \frac{M-2}{M} c_{2} \operatorname{vol}_{2} V$. This follows from the above inequality (5.22) and

$$
\begin{aligned}
\frac{M-1}{M} c_{2} \operatorname{vol}_{2} V \cdot h_{0} \leq \operatorname{vol}\left(Z_{m}\left(0, h_{0}\right) \cap \Omega^{\prime}\right) & =\int_{0}^{h_{0}} \int_{Q_{m}} \mathbb{1}_{Z_{m}\left(0, h_{0}\right) \cap \Omega^{\prime}}((\hat{\xi}, g(\hat{\xi}))+t v) \mathrm{d} \hat{\xi} \mathrm{d} t \\
& \leq h_{0} \cdot \sup _{t \in\left(0, h_{0}\right)} \operatorname{vol}_{2}\left(\left\{\hat{\xi}: \hat{\xi} \in Q_{m},(\hat{\xi}, g(\hat{\xi}))+t v \in Z_{m}\left(0, h_{0}\right) \cap \Omega^{\prime}\right\}\right),
\end{aligned}
$$

which implies the existence of such $H_{m,+}$. Analogously, there is an $H_{m,-} \in\left(0, h_{0}\right)$ such that

$$
P_{m}^{-}:=\left\{\hat{\xi}: \hat{\xi} \in Q_{m},(\hat{\xi}, g(\hat{\xi}))-H_{m,-} v \in Z_{m}\left(-h_{0}, 0\right) \cap \Omega^{\prime}\right\}
$$

has volume $\operatorname{vol}_{2} P_{m}^{-} \geq \frac{M-2}{M} c_{2} \operatorname{vol}_{2} V$. Now we set $P_{m}:=P_{m}^{+} \cap P_{m}^{-}$and obtain with the help of (5.17)

$$
\begin{aligned}
\operatorname{vol}_{2} P_{m} & =\operatorname{vol}_{2} P_{m}^{+}+\operatorname{vol}_{2} P_{m}^{-}-\operatorname{vol}_{2}\left(P_{m}^{+} \cup P_{m}^{-}\right) \geq \operatorname{vol}_{2} P_{m}^{+}+\operatorname{vol}_{2} P_{m}^{-}-\operatorname{vol}_{2} Q_{m} \\
& \geq 2 \frac{M-2}{M} c_{2} \operatorname{vol}_{2} V-c_{3} \operatorname{vol}_{2} V=\left(2 \frac{M-2}{M} c_{2}-c_{3}\right) \operatorname{vol}_{2} V
\end{aligned}
$$

We chose $c_{2}=\frac{3}{8}$ and $c_{3}=\frac{5}{8}$, thus by setting e.g. $M=13$ we obtain the estimate

$$
\operatorname{vol}_{2} P_{m} \geq \frac{1}{104} \operatorname{vol}_{2} V
$$

where the lower bound is independent of $m$. 
Step 4. In the estimate (5.18) of Step 2 we now set $h_{ \pm}:=H_{m, \pm}$ and arrive at

$$
\begin{aligned}
\int_{\Omega}\left|\nabla \varphi_{m}\right|^{p} \mathrm{~d} \xi \geq & \int_{Z_{m}\left(-H_{m,-}, H_{m,+}\right)}\left|\nabla \varphi_{m}\right|^{p} \mathrm{~d} \xi \\
\geq & \frac{1}{\left(H_{m,+}+H_{m,-}\right)^{p-1}} \int_{Q_{m}}\left|\varphi_{m}\left((\hat{\xi}, g(\hat{\xi}))+H_{m,+} v\right)-\varphi_{m}\left((\hat{\xi}, g(\hat{\xi}))-H_{m,-v}\right)\right|^{p} \mathrm{~d} \hat{\xi} \\
\geq & \frac{1}{\left(H_{m,+}+H_{m,-}\right)^{p-1}} \int_{P_{m}} \mid \varphi_{m}\left((\hat{\xi}, g(\hat{\xi}))+H_{m,+} v\right)-\varphi\left((\hat{\xi}, g(\hat{\xi}))+H_{m,+} v\right) \\
& \quad+\varphi\left((\hat{\xi}, g(\hat{\xi}))+H_{m,+} v\right)-\varphi\left((\hat{\xi}, g(\hat{\xi}))-H_{m,-} v\right) \\
& \quad+\varphi\left((\hat{\xi}, g(\hat{\xi}))-H_{m,-} v\right)-\left.\varphi_{m}\left((\hat{\xi}, g(\hat{\xi}))-H_{m,-} v\right)\right|^{p} \mathrm{~d} \hat{\xi}
\end{aligned}
$$

By construction we have for every $\hat{\xi} \in P_{m}$ the inclusion $(\hat{\xi}, g(\hat{\xi})) \pm H_{m, \pm} v \in \Omega^{\prime}$. Thus for all but finitely many $m$ we infer from the uniform convergence of $\varphi_{m} \rightarrow \varphi$ on $\Omega^{\prime}$ and (5.16)

$$
\left|\varphi_{m}\left((\hat{\xi}, g(\hat{\xi})) \pm H_{m, \pm} v\right)-\varphi\left((\hat{\xi}, g(\hat{\xi})) \pm H_{m, \pm} v\right)\right| \leq \frac{c_{1}}{4}
$$

for all $\hat{\xi} \in P_{m}$. Together with (5.16) we further deduce

$$
\begin{aligned}
\int_{\Omega}\left|\nabla \varphi_{m}\right|^{p} \mathrm{~d} \xi & \geq \frac{1}{\left(H_{m,+}+H_{m,-}\right)^{p-1}} \int_{P_{m}}\left(c_{1}-\frac{c_{1}}{4}-\frac{c_{1}}{4}\right)^{p} \mathrm{~d} \hat{\xi} \\
& =\frac{1}{\left(H_{m,+}+H_{m,-}\right)^{p-1}}\left(c_{1} / 2\right)^{p} \operatorname{vol}_{2} P_{m} \underset{(5.23)}{\geq} \frac{1}{104}\left(c_{1} / 2\right)^{p} \operatorname{vol}_{2} V \frac{1}{\left(2 h_{0}\right)^{p-1}}
\end{aligned}
$$

for all but finitely many $m$. But $h_{0}$ can be chosen arbitrarily small. Hence

$$
\liminf _{m \rightarrow \infty} \int_{\Omega}\left|\nabla \varphi_{m}\right|^{p} \mathrm{~d} \xi=\infty
$$

and the proof of the lemma is finished.

Lemma 5.8 ( $\Gamma$-lim inf-inequality). Let $\varphi \in \operatorname{SBV}^{p}\left(\Omega ; \mathbb{R}^{3}\right) \cap \operatorname{Kin}(\Omega ;$ Box $)$ be such that $S_{\varphi}$ is contained in a piecewise $\mathrm{C}^{1}$-hypersurface, and let $\left(\varphi_{k}\right)_{k}$ be a sequence in $\operatorname{SBV}^{p}\left(\Omega ; \mathbb{R}^{3}\right) \cap \operatorname{Kin}(\Omega ; \mathbf{B o x})$ that strongly converges to $\varphi$ in $\mathrm{L}^{1}\left(\Omega ; \mathbb{R}^{3}\right)$. Then

$$
\mathcal{F}_{\text {Hom }}^{\gamma}(\varphi) \leq \liminf _{k \rightarrow \infty} \mathcal{F}_{\varepsilon_{k}}^{\gamma}\left(\varphi_{k}\right)
$$

Proof. Assume first, that $\nu_{\varphi, 1}=\nu_{\varphi, 2}=0$ in $\mathcal{H}^{2}$-a.e. point of $S_{\varphi}$. In this case the assertion follows from Proposition 4.5 (wherein we set $M:=\operatorname{SBV}^{p}\left(\Omega ; \mathbb{R}^{3}\right), \phi(v):=|v|$ and $F:=0$ ).

In the remaining case where $\nu_{\varphi, 1} \neq 0$ or $\nu_{\varphi, 2} \neq 0$ on a subset of $S_{\varphi}$ of positive $\mathcal{H}^{2}$-measure it is $\mathcal{F}_{\text {Hom }}^{\gamma}(\varphi)=\infty$. Assume $\infty>\liminf _{k} \mathcal{F}_{\varepsilon_{k}}^{\gamma}\left(\varphi_{k}\right)$. Consequently, there holds

$$
\infty>\liminf _{k \rightarrow \infty} \mathcal{F}_{\varepsilon_{k}}^{\gamma}\left(\varphi_{k}\right)=\lim _{m \rightarrow \infty} \mathcal{F}_{\varepsilon_{k(m)}}^{\gamma}\left(\varphi_{k(m)}\right) \quad \text { and } \quad \varphi_{k(m)} \in \mathrm{W}^{1, p}\left(\Omega_{\varepsilon_{k(m)}}^{\gamma} ; \mathbb{R}^{3}\right) \cap \operatorname{Kin}(\Omega ; \mathbf{B o x})
$$

for an appropriate subsequence $(k(m))_{m}$. However, we are told by Lemma 5.7 that

$$
\liminf _{m \rightarrow \infty} \int_{\Omega}\left|\nabla \varphi_{k(m)}\right|^{p} \mathrm{~d} x=\infty
$$


and conclude with the help of condition $(W 2)$

$$
\infty>\liminf _{k \rightarrow \infty} \mathcal{F}_{\varepsilon_{k}}^{\gamma}\left(\varphi_{k}\right)=\lim _{m \rightarrow \infty} \mathcal{F}_{\varepsilon_{k(m)}}^{\gamma}\left(\varphi_{k(m)}\right) \geq \liminf _{m \rightarrow \infty} \int_{\Omega} \alpha_{1}\left|\nabla \varphi_{k(m)}\right|^{p}-\alpha_{2} \mathrm{~d} x=\infty,
$$

which provides a contradiction. Therefore, we must have $\infty=\liminf _{k} \mathcal{F}_{\varepsilon_{k}}^{\gamma}\left(\varphi_{k}\right)$ and again the $\Gamma$-lim inf-inequality holds.

The -limsup-statement of Theorem 5.2 is once more proved by using predeformations.

Lemma 5.9 ( $\Gamma$-limsup-inequality). Let $\varphi \in \operatorname{SBV}^{p}\left(\Omega ; \mathbb{R}^{3}\right) \cap \operatorname{Kin}(\Omega ; \mathbf{B o x})$ be such that $S_{\varphi}$ is contained in a piecewise $\mathrm{C}^{1}$-hypersurface $S$ and $\mathcal{F}_{\mathrm{Hom}}^{\gamma}(\varphi)<\infty$. Then there is a sequence $\left(\varphi_{k}\right)_{k}$ with $\varphi_{k} \in \mathrm{W}^{1, p}\left(\Omega_{\varepsilon_{k}}^{\gamma} ; \mathbb{R}^{3}\right) \cap$ $\operatorname{Kin}(\Omega ;$ Box $)$ satisfying

$$
\varphi_{k} \rightarrow \varphi \quad \text { in } \mathrm{L}^{1}\left(\Omega ; \mathbb{R}^{3}\right) \quad \text { and } \quad \lim _{k \rightarrow \infty} \mathcal{F}_{\varepsilon_{k}}^{\gamma}\left(\varphi_{k}\right)=\mathcal{F}_{\text {Hom }}^{\gamma}(\varphi)
$$

Proof. Since $\mathcal{F}_{\text {Hom }}^{\gamma}(\varphi)<\infty$ we must have $\nu_{\varphi, 1}=\nu_{\varphi, 2}=0$ in $\mathcal{H}^{2}$-a.e. point of $S_{\varphi}$. Hence, $\nu_{\varphi} \in\left\{e_{3},-e_{3}\right\}$ $\mathcal{H}^{2}$-a.e and from Remark 4.3 we deduce $\nu_{S} \in\left\{e_{3},-e_{3}\right\}$. Hence, $S$ is contained in the union of finitely many $x_{1} x_{2}$-parallel hyperplanes, say

$$
S \subseteq P=\left\{\left(\hat{x}, x_{3}\right) \in \Omega: x_{3} \in\left\{a_{1}, \ldots, a_{m}\right\}\right\}
$$

for $-a<a_{1}<\ldots<a_{m}<a$. One easily constructs a piecewise affine predeformation $\Phi_{k}: \Omega \rightarrow \Omega$ that:

- differs only in a small $\varepsilon_{k}$-neighborhood $\mathcal{T}_{k}$ of $P$ from the identity mapping,

- depends only on $x_{3}$,

- is such that it lifts the nearest hyperplane $\left\{\left(\hat{x}, x_{3}\right): x_{3}=\varepsilon_{k} z_{k, i}\right\}, z_{k, i} \in \mathbb{Z}$, onto $\left\{\left(\hat{x}, x_{3}\right): x_{3}=a_{i}\right\}$, $i \in\{1, \ldots, m\}$.

Such $\Phi_{k}$ admits an estimate like (5.5) uniformly on $\Omega$ with constants independent of $k$, cf. [24], Section 4.5.3. Once again we obtain a recovery sequence $\left(\varphi_{k}\right)_{k}$ by composition $\varphi_{k}=\varphi \circ \Phi_{k}$. Proposition 4.8 implies $\varphi_{k} \in$ $\operatorname{SBV}^{p}\left(\Omega ; \mathbb{R}^{3}\right) \cap \operatorname{Kin}(\Omega ; \mathbf{B o x})$ and

$$
S_{\varphi_{k}} \subseteq \Phi_{k}^{-1}(P)=\bigcup_{i=1}^{m}\left\{\left(\hat{x}, x_{3}\right) \in \Omega: x_{3}=\varepsilon_{k} z_{k, i}\right\} \subseteq \Gamma_{C, \varepsilon_{k}}^{\gamma},
$$

thus $\varphi_{k} \in \mathrm{W}^{1, p}\left(\Omega \backslash \Gamma_{C, \varepsilon_{k}}^{\gamma} ; \mathbb{R}^{3}\right)=\mathrm{W}^{1, p}\left(\Omega_{\varepsilon_{k}}^{\gamma} ; \mathbb{R}^{3}\right)$. The same arguments we used in the proof of Lemma 5.6 reveal that $\left(\varphi_{k}\right)_{k}$ is indeed a recovery sequence (again see [24], Sect. 4.5.3).

Acknowledgements. The author thanks the anonymous referees for their many helpful remarks and valuable suggestions.

\section{REFERENCES}

[1] R.A. Adams and J.J.F. Fournier, Sobolev spaces, Pure and Applied Mathematics (Amsterdam) 140. Elsevier/Academic Press, Amsterdam, 2nd edition (2003).

[2] L. Ambrosio, N. Fusco and D. Pallara, Functions of bounded variation and free discontinuity problems. Oxford Mathematical Monographs, Oxford University Press, Oxford (2000).

[3] M. Barchiesi and G. Dal Maso, Homogenization of fiber reinforced brittle materials: the extremal cases. SIAM J. Math. Anal. 41 (2009) 1874-1889.

[4] A. Braides, $\Gamma$-convergence for beginners, Oxford Lecture Series in Mathematics and its Applications 22. Oxford University Press, Oxford (2002).

[5] A. Braides and V. Chiadò Piat, Another brick in the wall, in Variational problems in materials science, Progr. Nonlinear Differential Equation Appl. 68, Birkhäuser, Basel (2006) 13-24. 
[6] A. Braides, A. Defranceschi and E. Vitali, Homogenization of free discontinuity problems. Arch. Rational Mech. Anal. 135 (1996) 297-356.

[7] P.G. Ciarlet, Mathematical elasticity. Three-dimensional elasticity I, Studies in Mathematics and its Applications 20. North-Holland Publishing Co., Amsterdam (1988).

[8] P.G. Ciarlet and J. Nečas, Injectivity and self-contact in nonlinear elasticity. Arch. Rational Mech. Anal. 97 (1987) $171-188$.

[9] G. Cortesani and R. Toader, A density result in SBV with respect to non-isotropic energies. Nonlinear Anal. 38 (1999) 585-604.

[10] G. Dal Maso, An introduction to $\Gamma$-convergence, Progress in Nonlinear Differential Equations and their Applications 8. Birkhäuser Boston Inc., Boston, MA (1993).

[11] G. Dal Maso and G. Lazzaroni, Quasistatic crack growth in finite elasticity with non-interpenetration. Ann. Inst. Henri Poincaré Anal. Non Linéaire 27 (2010) 257-290.

[12] G. Dal Maso and C.I. Zeppieri, Homogenization of fiber reinforced brittle materials: the intermediate case. Adv. Calc. Var. 3 (2010) 345-370.

[13] L.C. Evans and R.F. Gariepy, Measure theory and fine properties of functions. Studies in Advanced Mathematics, CRC Press, Boca Raton, FL (1992).

[14] G.A. Francfort and C.J. Larsen, Existence and convergence for quasi-static evolution in brittle fracture. Commun. Pure Appl. Math. 56 (2003) 1465-1500.

[15] A. Giacomini and M. Ponsiglione, Non-interpenetration of matter for SBV deformations of hyperelastic brittle materials. Proc. R. Soc. Edinb. Sect. A 138 (2008) 1019-1041.

[16] G.A. Iosif'yan, Homogenization of problems in the theory of elasticity with Signorini boundary conditions. Mat. Zametki $\mathbf{7 5}$ (2004) 818-833.

[17] N. Kikuchi and J.T. Oden, Contact problems in elasticity: a study of variational inequalities and finite element methods, SIAM Studies in Applied Mathematics 8. Society for Industrial and Applied Mathematics (SIAM), Philadelphia, PA (1988).

[18] D. Lukkassen, G. Nguetseng and P. Wall, Two-scale convergence. Int. J. Pure Appl. Math. 2 (2002) 35-86.

[19] A. Mikelić, M. Shillor and R. Tapiéro, Homogenization of an elastic material with inclusions in frictionless contact. Math. Comput. Model. 28 (1998) 287-307.

[20] O. Pantz, The modeling of deformable bodies with frictionless (self-)contacts. Arch. Ration. Mech. Anal. 188 (2008) $183-212$.

[21] L. Scardia, Damage as $\Gamma$-limit of microfractures in anti-plane linearized elasticity. Math. Models Methods Appl. Sci. 18 (2008) 1703-1740.

[22] L. Scardia, Damage as the $\Gamma$-limit of microfractures in linearized elasticity under the non-interpenetration constraint. Adv. Calc. Var. 3 (2010) 423-458.

[23] A. Signorini, Sopra alcune questioni di statica dei sistemi continui. Ann. Scuola Norm. Sup. Pisa Cl. Sci. 2 (1933) $231-251$.

[24] P.E. Stelzig, Homogenization of many-body structures subject to large deformations and noninterpenetration. Ph.D. Thesis, Technische Universität München (2009). Available electronically at http://nbn-resolving.de/urn/resolver.pl?urn:nbn:de: bvb:91-diss-20091214-797081-1-9.

[25] J.Y. Wong, Theory of ground vehicles. John Wiley \& Sons Inc., New York (2001). 